\title{
Microstructural Mechanics Model of Anisotropic-Thermal-Expansion-Induced Microcracking
}

\author{
Narayanaswamy Sridhar, Wuhua Yang, and David J. Srolovitz \\ Department of Materials Science and Engineering, University of Michigan, \\ Ann Arbor, Michigan 48109
}

Edwin R. Fuller, Jr. Materials Science and Engineering Laboratory, National Institute of Standards and Technology, Gaithersburg, Maryland 20899

\begin{abstract}
Thermal-expansion-induced microcracking in single-phase ceramics has been simulated using a simple mechanics model based upon a regular lattice of brittle, elastic springs. Microcracks preferentially form at grain boundaries and propagate either into the bulk or along grain boundaries, depending on the toughness of the boundaries relative to the grain interiors. The present results show that anisotropic-thermal-expansion-induced microcracking can be more severe for either large or small grain size samples depending on the damage measure employed. At very small misfit strains, the large grain microstructure develops microcracks before the small grain microstructure. However, over most of the misfit strain regime examined, the total length/area of all cracks in a sample is larger when the grain size is small. This is manifested in a larger decrement of the elastic modulus in small grain size samples as compared with large grain size samples at the same misfit $(\Delta T)$. However, large grain sizes are more detrimental with regard to fracture properties. This is because the fracture stress scales as inversely with the crack length and large grain samples exhibit larger microcracks than small grain samples. Unlike in the unconstrained samples, when a sample is constrained during a temperature excursion, the stress created by the overall thermal expansion can directly lead to fracture of the entire sample.
\end{abstract}

\section{Introduction}

$\mathbf{M}$ ANY ceramic materials are known to undergo spontaneous cracking when cooled from high processing temperatures. The presence of microcracks modifies several physical properties including thermal diffusivity, dielectric constant, acoustic transparency, and elastic moduli.' Microcracking can also lead to an increase in fracture toughness, ${ }^{2}$ presumably associated with the microcracking-induced dilatation and also partly due to the formation of a process zone ahead of a propagating crack. ${ }^{3,4}$ However, the contribution of microcracking to toughening is usually minor. ${ }^{5}$ The tendency to form these cracks is known to increase with increasing temperature excursions and increasing grain size $e^{6}$ and is often attributable to residual stresses that develop from either thermal contraction anisotropy or non-shape-preserving phase transformations. ${ }^{7}$ In multiphase

T. Michalske-contributing editor

\footnotetext{
Manuscript No. 194300. Received August 16, 1993; approved December 20, 1993 Support for S.N. was provided by the National Institute of Standards and Technology through Grant No. 70NANB1H1147; support for W.Y. and D.J.S. was provided by the U.S. Air Force Office of Scientific Research through Grant No. AFOSR-90-0141.
}

materials, thermal-expansion-induced microcracking may also result from the difference in the coefficient of thermal expansion of the different phases. In single-phase, polycrystalline materials, however, thermal-expansion-induced microcracking is associated with the crystalline anisotropy of the coefficient of thermal expansion. Since many single-phase, ceramic materials are neither isotropic nor cubic, thermal expansion anisotropy is thought to be the dominant cause of microcracking associated with temperature excursions. This paper focuses on the role of anisotropic thermal expansion in microcracking.

Thermal-expansion-anisotropy-induced microcracking has received a great deal of experimental ${ }^{2.5,8,9}$ and theoretical ${ }^{3,4,10,11}$ attention. A fracture mechanics analysis of microcracking ${ }^{10,11}$ reveals an essential dependence of microcracking on microstructural dimensions. In particular, dimensional considerations dictate that microcracks initiate when grains exceed a critical size. Obtaining an exact solution to this problem is a formidable task, since this involves adding the effects of thermal expansion anisotropy to the tensor Hooke's law and then integrating over the temperature range of interest to yield the thermal-expansion-induced stresses. This procedure should be applied to nonuniform microstructures in order to obtain a realistic stress distribution. Therefore, most of the theoretical analyses of anisotropic-thermal-expansion-induced microcracking have made certain simplifying assumptions such as the presence of very idealized microstructures (e.g., Refs. 4, 10, 11, and 12). These include pairs of grains, hexagonal arrays of grains, etc. Real microstructures, on the other hand, exhibit a wide distribution of microstructural geometries. ${ }^{13}$ This is particularly important since fracture properties are determined by the extremes in the local (microstructure-dependent) stresses rather than their average values. Thus, an understanding of microcracking-related phenomena requires a realistic, microstructure-based description of microcracked microstructures.

In this paper, we examine thermal-expansion-anisotropyinduced microcracking based upon a simple mechanical model which is solved numerically. ${ }^{14}$ We refer to this model as the "microstructural mechanics model" because it is capable of describing the stress distribution and fracture behavior of mate rials with arbitrarily complex microstructure. We employed the microstructural mechanics model to study microcracking in a realistic polycrystalline microstructure as a function of grain size, thermal expansion anisotropy, and relative grain boundary to bulk toughness, and to determine the effect of external stress on microcracking.

\section{Microstructural Mechanics Model}

\section{(I) Basic Model}

The mechanics model employed in the simulations, described below, is based upon the elastic properties of a network of springs. Rather than discretizing the equations of elasticity, the elastic continuum has been replaced with a lattice of 
springs. The model consists of a triangular array of lattice points which are connected by bonds, as indicated in Fig. 1. The energy, $E$, of this array of bonds is the sum of a bond stretching energy, $\Phi$, and a bond bending energy, $\Psi$, and is given by ${ }^{14}$

$$
E=\frac{1}{2} \sum_{i=1}^{N} \sum_{j}^{\mathrm{nn}} \Phi_{i j}\left(R_{i j}\right)+\frac{1}{6} \sum_{i=1}^{N} \sum_{j} \sum_{k} \Psi_{i j k}\left(\mathbf{R}_{i j}, \mathbf{R}_{i k}\right)
$$

where the sums on $i$ are over all $N$ sites in the system, the sums on $j$ are over all sites which are nearest neighbors (nn) of site $i$, the sum on $k$ is over all sites that are nearest neighbors of sites $i$ and $j$, and $\mathbf{R}_{i j}$ is the vector separation of sites $i$ and $j$ of magnitude $R_{i i}$. The bond stretching $(\Phi)$ and bending $(\Psi)$ functions may be chosen to represent linear elastic (stretching) springs and watch (bending) springs, respectively, and may be expressed as ${ }^{14}$

$$
\begin{aligned}
& \phi_{i j}=\frac{1}{2} k_{i j}\left(R_{i j}-a_{0}\right)^{2} \\
& \Psi_{i j k}=\frac{1}{2} c_{i j k}\left(\frac{\vec{R}_{i j} \cdot \vec{R}_{i k}}{\left|\vec{R}_{i j}\right|\left|\vec{R}_{i k}\right|}-b_{0}\right)^{2}
\end{aligned}
$$

where $k_{i j}$ and $c_{i j k}$ are constants that scale the stiffness of the bond stretching and bond bending interactions, and $a_{0}$ and $b_{0}$ are related to the equilibrium bond lengths and bond angles, respectively.

For a uniform triangular lattice of springs, all of the $k_{i j}$ and $c_{i j k}$ are identical $\left(k_{i j}=k\right.$ and $\left.c_{i j k}=c\right)$. In this case, the nonzero elastic constants (in Voigt notation) are given by

$$
\begin{aligned}
& C_{11}=C_{22}=\frac{9}{8} k a_{0}^{2}+\frac{21}{16} c \\
& C_{33}=\frac{3}{8} k a_{0}^{2}+\frac{21}{16} c \\
& C_{12}=\frac{3}{8} k a_{0}^{2}+\frac{21}{16} c
\end{aligned}
$$

The symmetry of the elastic constant tensor is that of an isotropic continuum. As in an isotropic solid, there are only two independent elastic constants and the symmetry is that of the triangular lattice (see, e.g., Ref. 15). Consequently, the Lamé constant $\lambda$, shear modulus $\mu$, Poisson's ratio $\nu$, Young's modulus $E$, and bulk modulus $B$ may also be written in terms of the $C_{i j}$ or, alternatively, $c$ and $k$ as

$$
\begin{aligned}
& \mu=C_{33}=\frac{1}{2}\left(C_{11}-C_{12}\right)=\frac{3}{8} k a_{0}^{2}+\frac{21}{16} c \\
& \nu=C_{12} / C_{11}=\frac{2 k a_{0}^{2}-7 c}{6 k a_{0}^{2}+7 c} \\
& E=2 \mu(1+\nu)=3 k a_{0}^{2} \frac{2 k a_{0}^{2}+7 c}{6 k a_{0}^{2}+7 c} \\
& B=\frac{E}{3(1-2 v)}=k a_{0}^{2} \frac{2 k a_{0}^{2}+7 c}{2 k a_{0}^{2}+21 c}
\end{aligned}
$$

The $\alpha \beta$ component of the stress at site $i$ in the model may be written as

$\sigma_{i}^{\alpha \beta}=\frac{1}{2 \Omega} \sum_{j}\left(\frac{\partial \Phi_{i j}}{\partial R_{i j}} \frac{\partial R_{i j}}{\partial R_{i j}^{\beta}} R_{i j}^{\alpha}+\sum_{k}\left[\frac{\partial \Psi_{i j}}{\partial R_{i j}} \frac{\partial R_{i j}}{\partial R_{i j}^{\beta}} R_{i j}^{\alpha}+\frac{\partial \Psi_{i j}}{\partial R_{i j}} \frac{\partial R_{i j}}{\partial R_{i j}^{\alpha}} R_{i j}^{\beta}\right]\right)$

where $\Omega$ is the area associated with site $i, \alpha$ and $\beta$ are Cartesian directions that take on the values $x$ and $y$ (or 1 and 2), and $R_{i j}^{a}$ is the $\alpha$ component of the distance between sites $i$ and $j$. The macroscopic stress in the sample is obtained by averaging $\sigma_{i}^{\alpha \beta}$ over all sites.

Microstructural features are incorporated into the model by associating different properties with each bond. For example, a polycrystalline body is simulated by identifying a bond as a bulk bond if it separates two adjacent lattice sites within the same grain or as a grain boundary bond if it separates two adjacent lattice sites belonging to different grains. The fracture properties of the model are incorporated via a bond-typedependent failure criterion. In the present study, a bond breaks irreversibly when its strain energy

$$
E\left({ }_{i j}\right)=\Phi_{i j}\left(R_{i j}\right)+\frac{1}{2} \sum_{k} \Psi_{i j k}\left(\mathbf{R}_{i j}, \mathbf{R}_{i k}\right)
$$

exceeds a critical strain energy $E_{i j}^{\mathrm{c}}$, where the magnitude of $E^{\mathrm{c}}$ is different for bulk and grain boundary bonds. A multiphase material may be modeled by choosing the elastic parameters $k_{i}$ and $c_{i j k}$ to depend on the phases of sites $i, j$, and $k$. Misfit or eigen strain may be included by choosing the constants $a_{0}$ and $b_{0}$ to also be site dependent (i.e., $a_{i j}$ and $b_{i j k}$ ).

While this discreteness of the present model does introduce an artificial length scale (the lattice parameter $a_{0}$ ) into the simulations, it also provides a simple mechanism for incorporating a

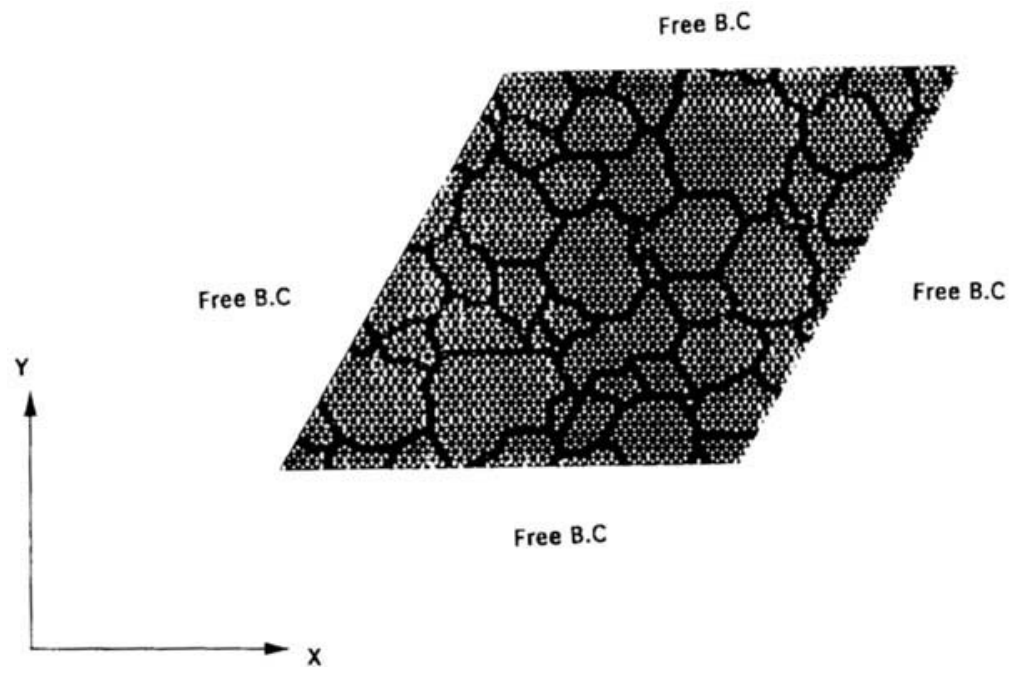

Fig. 1. Polycrystalline microstructure mapped onto a triangular lattice of brittle, linear elastic springs. 
wide variety of microstructural effects, as described below. This simple model retains most of the main features and trends found in continuum elastic models; however, the results should not be viewed as quantitative.

\section{(2) Polycrystalline Microstructure}

In order to simulate fracture in a polycrystalline material, a realistic polycrystalline microstructure was first mapped onto the two-dimensional, triangular lattice of the microstructural mechanics model. Such a polycrystalline microstructure was produced using the Monte Carlo simulation procedure introduced by Srolovitz and co-workers. ${ }^{16,17}$ This procedure has been shown to produce microstructures with grain size and grain topology distributions in excellent agreement with experiment. ${ }^{16,17}$

In short, a continuum microstructure is mapped onto a twodimensional triangular lattice containing $N=10000$ sites. Each lattice site is assigned a number, $S_{i}$, which corresponds to the orientation of the grain in which it is embedded. The number of distinct grain orientations is $Q$. Lattice sites which are adjacent to neighboring sites having different grain orientations are regarded as being adjacent to a grain boundary, while a site surrounded by sites with the same grain orientation is in the bulk or grain interior. The grain boundary energy is specified by associating a positive energy with grain boundary sites and zero energy for sites in the grain interior, according to the Potts Hamiltonian

$$
E=J \sum_{i=1}^{N} \sum_{i}^{\ln (i)}\left(1-\delta_{S_{i} s_{j}}\right)
$$

where $\delta_{i j}$ is the Kronecker delta and $J$ is a positive constant that sets the energy scale of the simulation. The kinetics of boundary motion are simulated via a zero-temperature Monte Carlo technique in which a lattice site is selected at random and its orientation is randomly changed to one of the other grain orientations. The change in energy associated with the change in orientation is then evaluated. If the change in energy is less than or equal to zero, the reorientation is accepted. However, if the energy is raised, the reorientation is rejected.

The microstructures were produced by initially assigning a random value of the grain orientation to each site $\left(1 \leq S_{i} \leq Q\right)$ and then running the Monte Carlo simulation procedure until the desired grain size was produced. The resultant two-dimensional polycrystalline microstructures are in good agreement with those found from taking cross sections through threedimensional polycrystalline materials and three-dimensional simulations. ${ }^{16,17}$ In addition to producing an accurate representation of observed microstructures, this simulation procedure has the advantage of producing microstructures on exactly the same lattice as that employed for the microstructural mechanics simulations.

\section{(4) Simulation Procedure}

The basic features of the present simulation technique employed in this study were described previously ${ }^{14}$ in an application in which the intergranular/transgranular transition was examined using strain-controlled tensile tests. Thermal expansion is incorporated by allowing the equilibrium lattice parameter, $a_{0}$ (see Eq. $(2 a)$ ), to scale with the change in temperature $\Delta T$ by an amount $a_{0}^{\text {new }}=a_{0}^{\text {old }}(1+\alpha \Delta T)$, where $\alpha$ is the coefficient of thermal expansion. The thermal contraction $\alpha \Delta T$ that results from cooling is referred to as the misfit strain $e$ and is equal to the change in the value of $a_{0}: e=-\left(a_{0}^{\text {ncw }}-a_{0}^{\text {old }}\right) / a_{0}^{\text {old }}$, where the sign was chosen such that positive $e$ corresponds to a contraction.

The present study focuses on anisotropic thermal expansion. The simplest method to include thermal expansion anisotropy is to make direct use of the inherent lattice nature of the simulation. As the present simulations are performed upon a triangular lattice, we choose one of the three inequivalent lattice directions to be the high thermal expansion direction and the other two directions to be low expansion directions. Since each grain in a microstructure has a well-defined crystallographic orientation, we included anisotropy by randomly choosing the high expansion direction from the three possible directions for each grain (i.e., for all of the sites within the grain) and then applying the prescribed misfit strain in that direction. One of the limitations of this approach is that it leads to only three types of anisotropic grain orientations whereas in a real polycrystalline material the direction of maximum expansion varies continuously with the crystallographic orientation of the grains. For the sake of convenience, the coefficients of thermal expansion for the low expansion orientations were set to zero. The application of misfit strains (contractions), as described above, corresponds to cooling a solid through a nonzero $\Delta T$. Unless the microstructure consists of a single crystal, this type of anisotropic thermal expansion will lead to an internal stress distribution within the solid that varies with location on a length scale comparable with the grain size.

The present simulations of anisotropic thermal expansion (contraction) induced microcracking were performed in the following manner: (1) a polycrystalline microstructure is produced using the Monte Carlo model; (2) prescribed values of the bulk $\left(E_{\mathrm{b}}^{\mathrm{c}}\right)$ and grain boundary $\left(E_{\mathrm{gb}}^{\mathrm{c}}\right)$ critical (fracture) energies are assigned to the appropriate bonds; (3) the high thermal expansion directions are assigned to each grain; (4) a small, finite misfit strain (contraction) increment is applied to the bonds oriented in the high expansion directions; (5) the total energy of the system is minimized with respect to the site coordinates using a (double precision) conjugate gradient algorithm; (6) the bond with the maximum energy ratio $E_{i j} / E_{i j}^{\mathrm{c}}$ is identified; (7) if this bond energy ratio is greater than unity, this bond is irreversibly broken and we return to step (5); otherwise, (8) if no bonds are broken during step 7 , we return to step 4 .

The present simulations were performed on lattices consisting of $10^{4}(100 \times 100)$ nodes. In order to model a sample of finite size, all four edges of the model were left free, resulting in a total of 29601 individual bonds. This lattice size was chosen such that the simulations could be performed in reasonable computational time on a 16 Mbyte computer workstation. In order to ensure that the results would be statistically significant, at least six simulations were performed, for each set of conditions, with different microstructures of identical physical descriptors (i.e., grain size, $E_{\mathrm{gb}}^{\mathrm{c}}, E_{\mathrm{b}}^{\mathrm{c}}$ ). The following parameters were employed: $k=1, c=(1 / 7), a_{0}=1, b_{0}=1 / 2$, and $E_{\mathrm{b}}^{\mathrm{c}}=$ $0.5 \times 10^{-4}$. The grain size $r$ (in units of $a_{0}$ ), the grain boundary critical energy $E_{\mathrm{gb}}^{\mathrm{c}}$ (and equivalently, the ratio $R=E_{\mathrm{gb}}^{\mathrm{c}} / E_{\mathrm{b}}^{\mathrm{c}}$ ), and the anisotropic-thermal-expansion-induced misfit strain $e$ were varied in the present study.

\section{Results}

\section{(1) Unconstrained Microcracking}

The first study focuses on the microcracking behavior of a polycrystalline solid with a traction free surface during cooling. Since no external loads or constraints are applied to the solid, it is free to contract. Although the surfaces are traction free, internal residual stresses are generated as a result of the anisotropy in the thermal expansion coefficients.

(A) Tough Grain Boundaries: The first series of simulations were run for the case where the grain boundary has the same critical energy or strength as the bulk $\left(R=E_{\mathrm{gb}}^{\mathrm{c}} / E_{\mathrm{b}}^{\mathrm{c}}=\right.$ 1.0 ). Since the grain boundary has the same strength as the bulk, the grain boundary is simply the location where the direction of maximum thermal expansion changes. Figure 2 shows a series of microstructures with broken bonds indicated as a function of the misfit strain $e$ for a mean grain size of 14.4. At a misfit strain of $e=0.0120$ (Fig. 2(a)), few bonds are broken. Most of these cracks (groups of broken bonds) have nucleated at sites near the grain boundaries. Some of these cracks grow into (and through) the grain interior, such as the one near the center of Fig. 2(a). On further increase of the misfit strain (to $e=0.0150$ ), we 


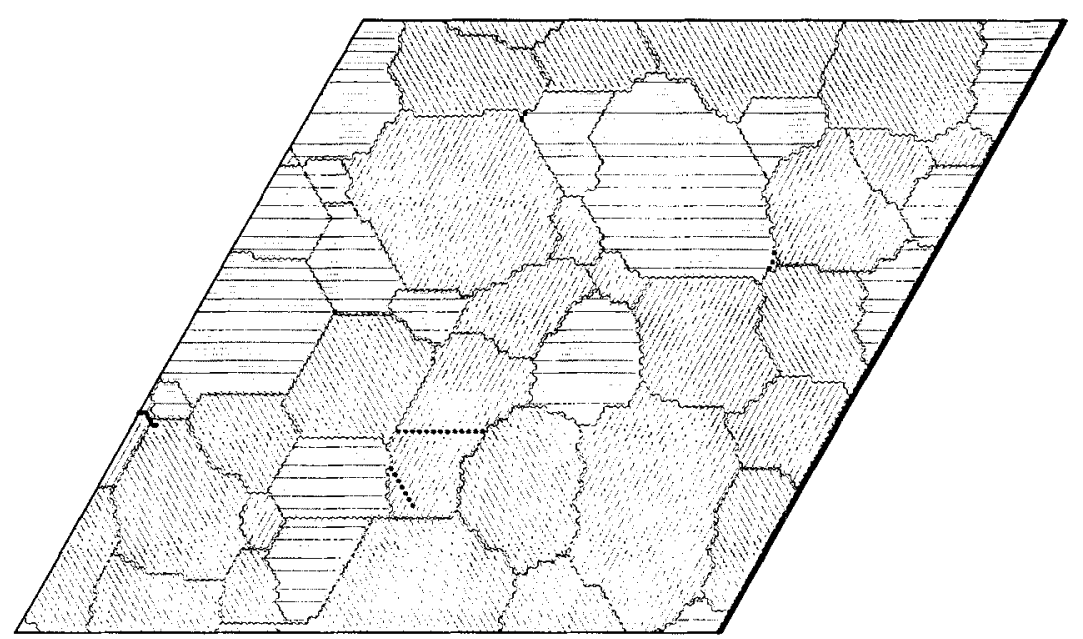

(a)

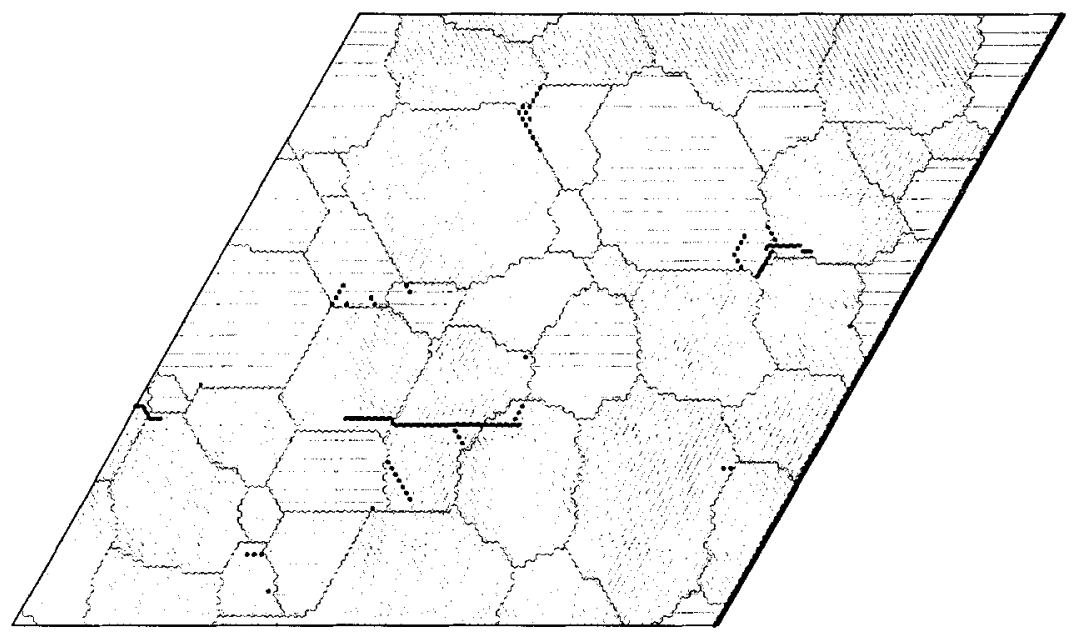

(b)

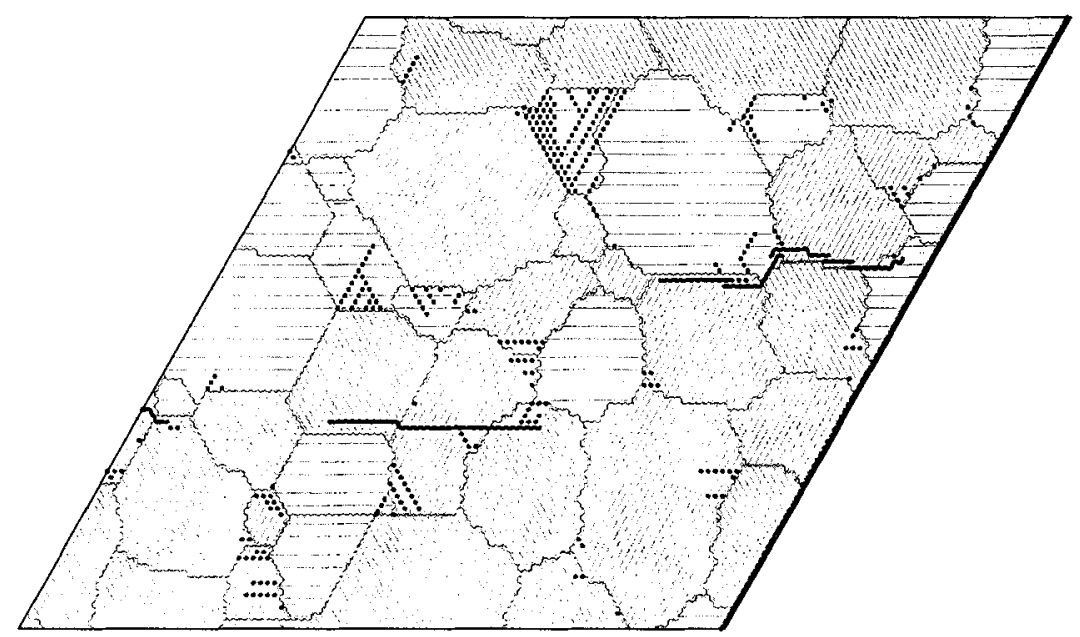

(c)

Fig. 2. Microcracked polycrystalline microstructures for a series of increasing misfit strains: (a) $e=0.0120$, (b) $e=0.015$, and (c) $e=0.0195$. The grain size is $r=14.4$ (in units of the unstrained spring length) and the ratio of grain boundary to grain interior toughness is $R=1.0$. Light solid lines outline the periphery of each grain and the horizontal lines within each grain indicate the orientation of the high thermal expansion axis. The dark lines indicate the location of broken bonds.

observe that new cracks continue to be nucleated at sites near the grain boundaries (Fig. 2(b)). Several of the cracks that were formed at lower misfit strains grow into large continuous cracks. These cracks have lengths comparable to the grain size. A further increase in the misfit strain (to $e=0.0195$ ) shows a large increase in the microcrack density (Fig. 2(c)). New cracks have nucleated and some of the smaller cracks have grown into larger continuous cracks. Some grains are microcrack free whereas others contain a large density of microcracks. A plot of the total number of broken bonds $\rho_{c}$ as a function of the misfit 
strain (Fig. 3) shows that $\rho_{c}$ increases with misfit strain (e) in a nonlinear manner.

The dependence of the microcracking behavior on grain size was investigated in order to examine the experimentally observed grain size effect in anisotropic-thermal-expansioninduced damage. Microcracked microstructures of three different grain sizes $(4.5,14.4$, and 19.8) are shown in Fig. 4 at a misfit strain of $e=0.012$. In the smallest grain size sample (Fig. 4(a)), we find only two broken bonds at this strain. The intermediate grain size sample (Fig. 4(b)) shows substantially more $(36)$ broken bonds at this strain level. The microcracks nucleated at the grain boundaries or at sites very close to the boundaries and, in one case, has propagated completely through a grain. In the largest grain size $(r=19.8)$ microstructure (Fig. 4(c)), the number of broken bonds is large and the microstructure contains cracks of length comparable to the larger grain size. Figure 3 summarizes the broken bond density $\left(\rho_{c}\right)$ dependence on grain size and on misfit strain. The inset in Fig. 3 shows the small misfit strain region in more detail. Microcrack initiation occurs at $e=0.0092$ for the largest grain size sample whereas it occurs at a $50 \%$ larger strain $(e=0.014)$ for the smallest grain size sample. In all cases, microcracking begins at a lower misfit strain the larger the grain size. This is in accordance with earlier experimental observations and analyses.

However, on increasing the misfit strains still further (to $e=$ 0.0195), the microstructures (Fig. 5) exhibit the opposite trend; i.e., small grain size samples contain more broken bonds than do large grain size samples (see Fig. 3). In the small grain size sample (Fig. 5(a)), the number of microcracks is very large, they are distributed throughout the sample, and they are shortof order the small grain size. As the grain size gets larger (Fig. 5(b)), we observe many fewer microcracks; however, these cracks tend to be longer-again, of order the grain size. This trend continues for the largest grain size microstructure (Fig. 5(c)), where the number of microcracks is even smaller, but they are longer. In all cases, the microcracks are initiated at the grain boundaries and propagate over length scales comparable to the grain size. Some grains appear to be crack free, suggesting the importance of local grain orientation relative to their neighborhood.

(B) Weak Grain Boundaries: When the grain boundaries are less tough than the grain interiors $(R<1)$, the microcracking behavior is significantly modified. The present set of simulations were performed for the case where the critical energies to break a bond traversing the grain boundary were $4 / 10$ that of a bond inside a grain $(R=0.4)$. This is equivalent to a ratio of the critical strain energy release rates $\left(G_{c}\right)$ of 0.4 . In these simulations, the grain boundary both is a weak (easy) path for crack propagation and serves as a position where the orientation of the misfit changes. $R=0.4$ was chosen because it was close to the $R$ value of the intergranular-transgranular fracture transition found in earlier simulations ${ }^{14}$ under uniaxial tension.

Figures 6(a) to (c) show the spatial distribution of microcracks for several different anisotropic-thermal-expansioninduced misfit strains in a microstructure with a mean grain size of $r=14.4$. As in the $R=1$ case (see Fig. 2), the initial microcracks (Fig. 6(a), $e=0.0095$ ) form along the grain boundaries and points where three grain boundaries meet (i.e., triple points). However, unlike in the $R=1$ case, the microcracks propagate preferentially along the grain boundries. Increases in misfit strain (Figs. 6(b) and (c)) lead to the nucleation of additional microcracks along the grain boundary and the extension of the cracks, formed at lower $e$, along the grain boundaries. The cracks tend to grow to lengths comparable with the grain size, as in the $R=1$ case. Comparing the microcracking behavior of the tough grain boundary and weak grain boundary cases at the same grain size and value of misfit strain (cf. Figs. 2(a) and $6(\mathrm{~b})$ ), we note that the weak grain boundary microstructure has more broken bonds. This is seen more quantitatively by directly comparing the numbers of broken bonds $\left(\rho_{c}\right)$ in the $R=1$ (Fig. 3) and $R=0.4$ (Fig. 7) cases. The main distinction between these two plots is the observation that microcracking is initiated at a lower value of misfit strain for $R=0.4$ than for $R=1.0$.

Figure 8 shows the effect of grain size on the spatial distribution of microcracks at small misfit strains and for weak grain boundaries $(R=0.4)$. Just as in the case of the tough grain boundaries, increasing grain size at fixed misfit leads to an increase in the number of broken bonds. As the grain size increases, the number of microcracks decreases, but the lengths of the microcracks increase such that $\rho_{c}$ (which is the product of the number of microcracks and their length) increases. Unlike in the tough grain boundary $(R=1)$ case, microcracks both form and propagate solely along the grain boundaries when they are weak $(R=0.4)$. The inset in Fig. 7 shows that microcracks form at smaller misfit strains when the grain size is large than when it is small. Microcrack initiation occurs at $e=$ 0.0075 for the largest grain size $(r=19.8)$ sample, whereas it occurs only at $e=0.0082$ for the smallest grain size $(r=4.5)$ sample. This dependence of microcracking behavior on grain size is similar to that observed in the strong grain boundary case (above).

At a higher misfit strain ( $e=0.0195)$, the smallest grain size microstructure (Fig. 9(a)) has the largest number of broken

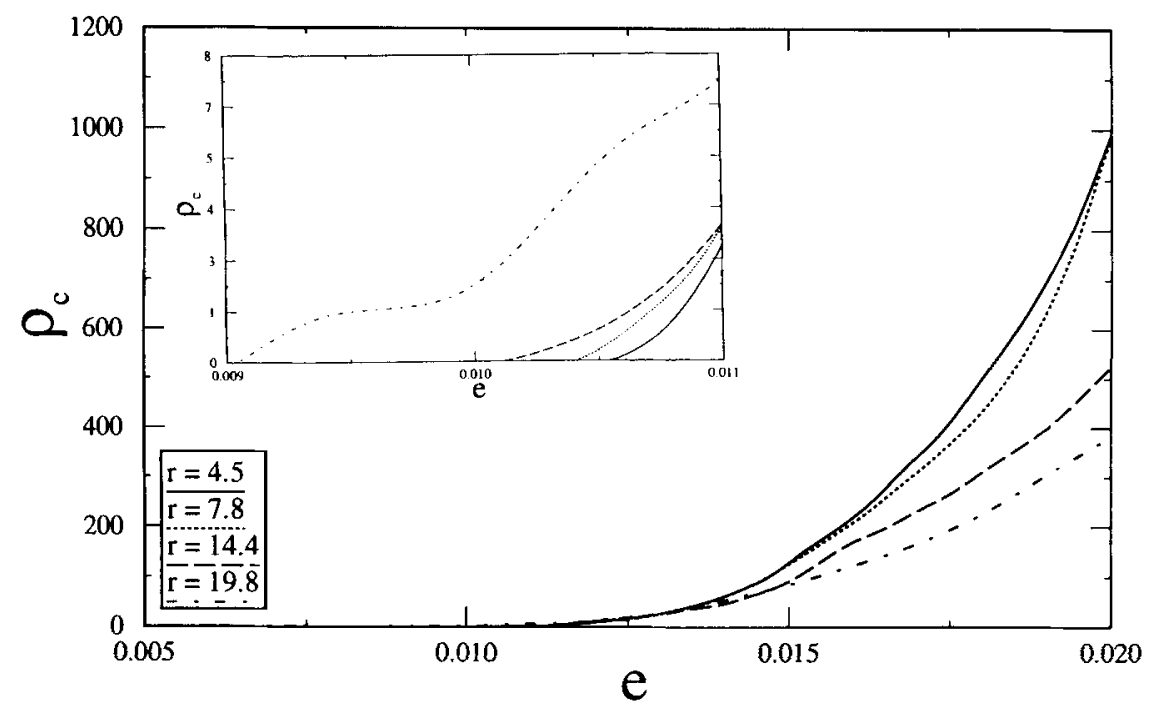

Fig. 3. Total number of broken bonds in the model $\rho_{\mathrm{c}}$ as a function of misfit strain $e$ for several different grain sizes in microstructures for which the grain boundary and bulk toughnesses are equal, $R=1.0$. The inset shows the small misfit strain data in more detail. 


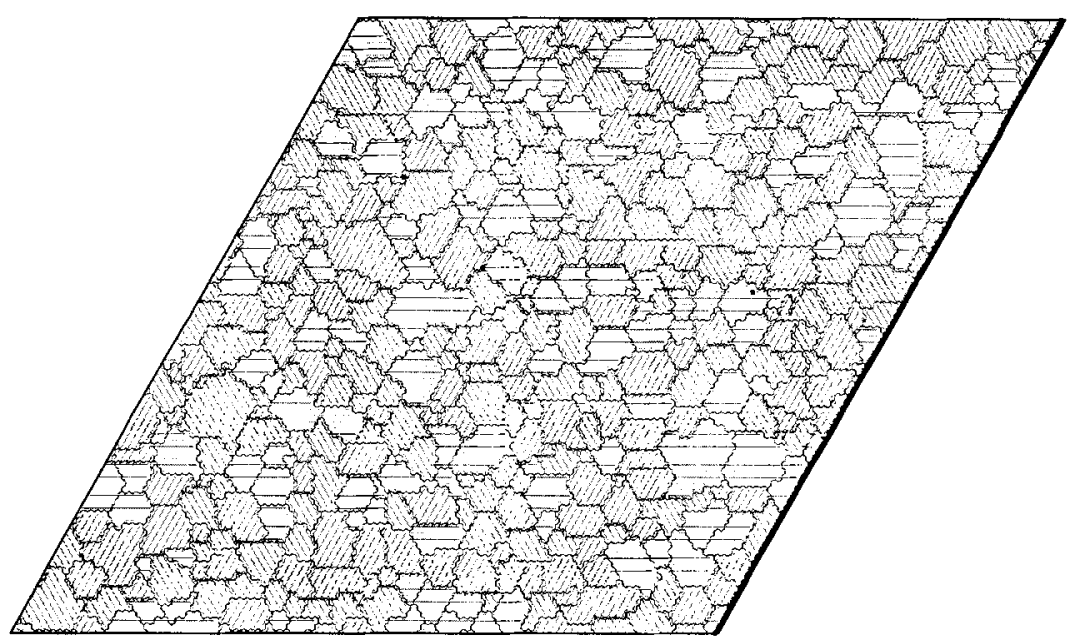

(a)

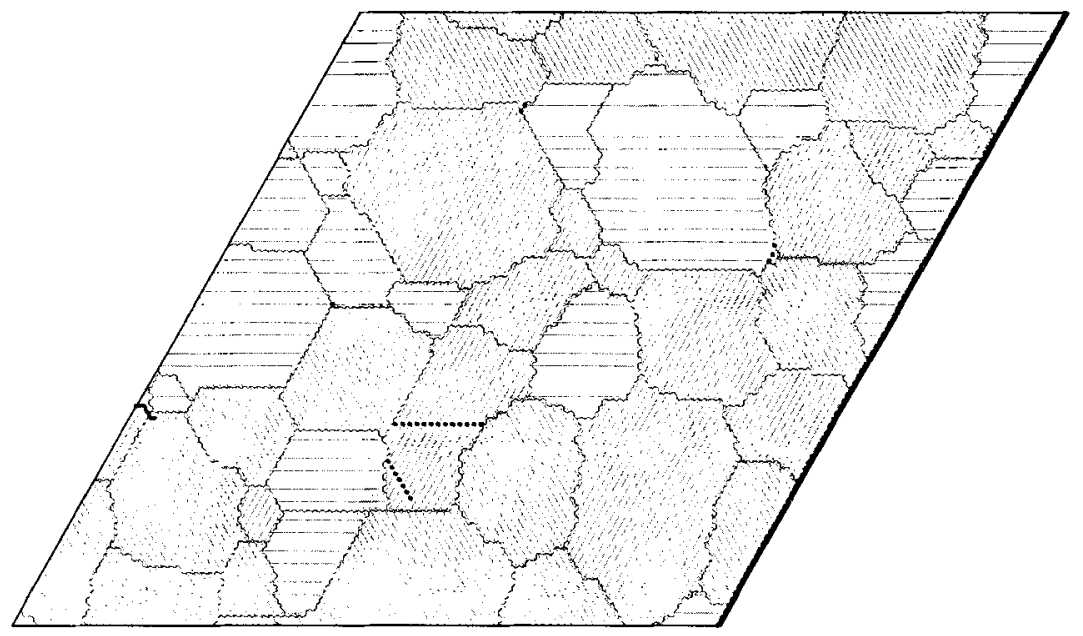

(b)

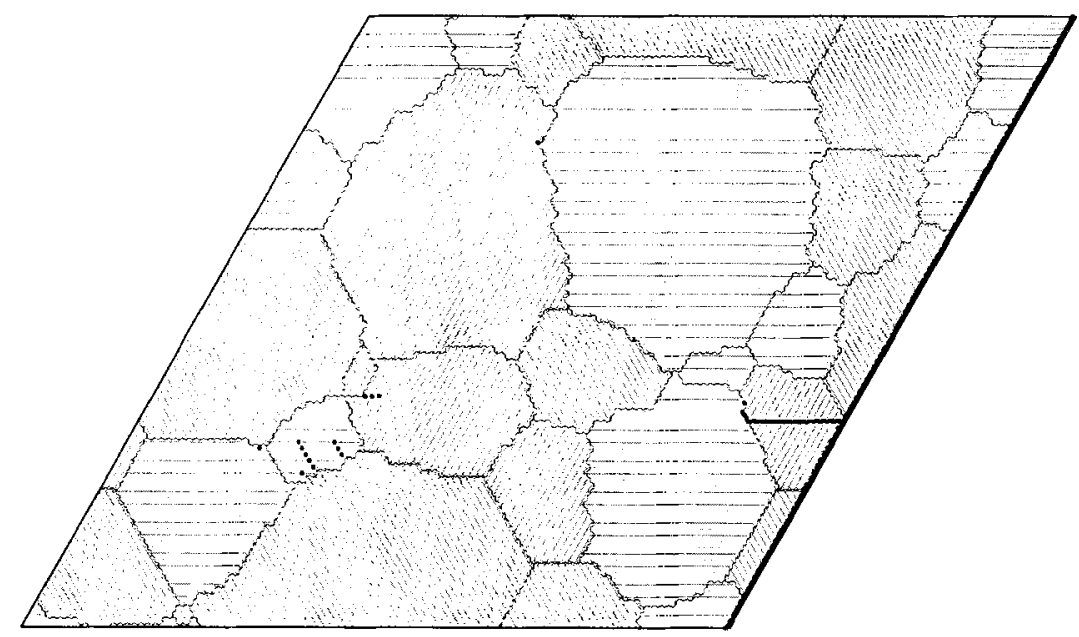

(c)

Fig. 4. Microcracked polycrystalline microstructures for a series of grain sizes: (a) $r=4.5$, (b) $r=14.4$, and (c) $r=19.8$. These results are for a small misfit strain $(e=0.0120)$ and the ratio of grain boundary to grain interior toughness is $R=1.0$.

bonds in the form of many, widely distributed short cracks. On the other hand, larger grain size samples (Figs. 9(b) and (c)) exhibit a smaller number of microcracks, but of substantially larger extent. Therefore, just as in the tough boundary case discussed above, $\rho_{c}$ is larger for larger grain samples at small misfit and larger for small grain samples at large misfit (see Fig. 7).

\section{(2) Constrained Microcracking}

The above results were obtained on a polycrystalline solid of finite extent that was free to expand or contract in both directions (i.e., it had free surfaces). This section reports on anisotropic-thermal-expansion-induced microcracking in a solid body that is rigidly constrained at two opposing surfaces, as if 


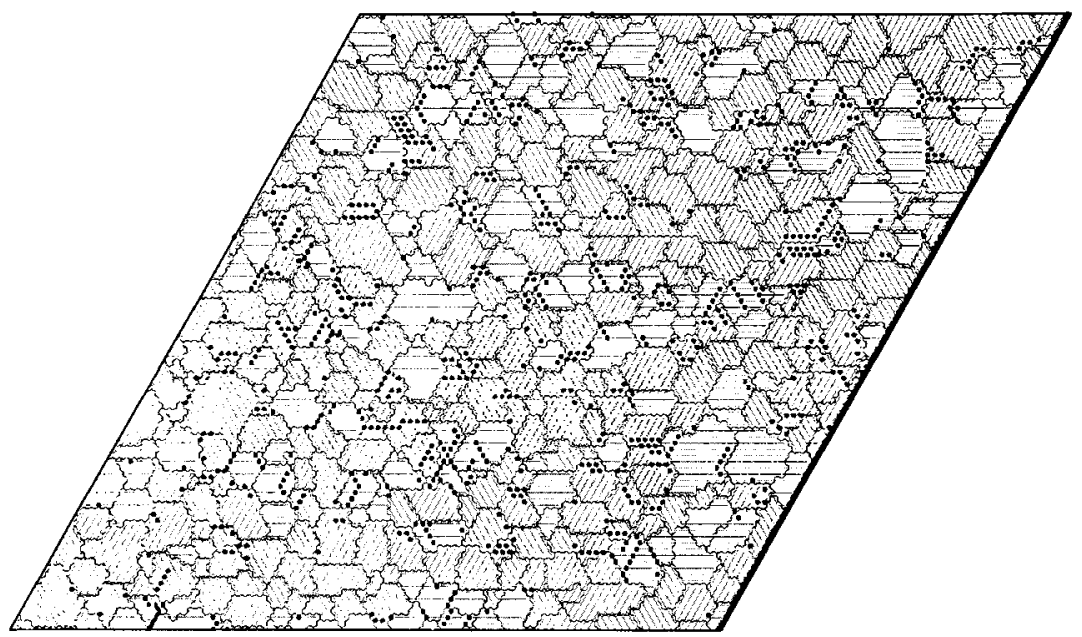

(a)

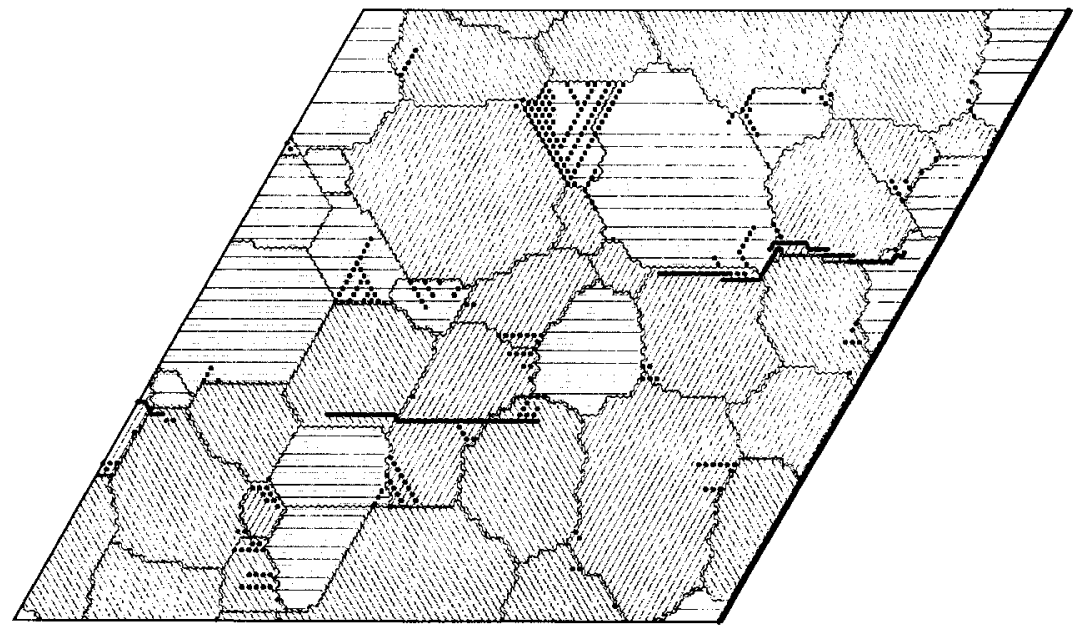

(b)

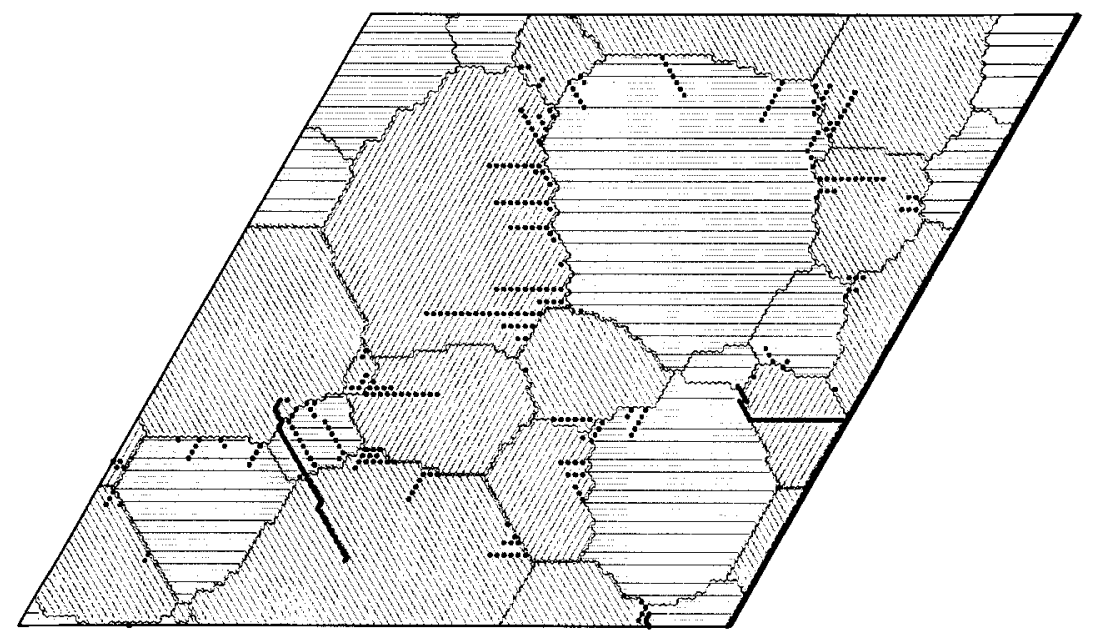

(c)

Fig. 5. Microcracked polycrystalline microstructures for a series of grain sizes: (a) $r=4.5$, (b) $r=14.4$, and (c) $r=19.8$. These results are for a large misfit strain $(e=0.0195)$ and the ratio of grain boundary to grain interior toughness is $R=1.0$.

held between the grips of a perfectly rigid testing machine. Free surfaces were maintained in one direction and periodic boundary conditions in the other. Although at $\Delta T=0$ there is no external stress, the application of the misfit strain (contraction obtained by cooling) results in a net tensile stress across the sample (parallel to the constraint direction) which is superimposed on the nonuniform, anisotropic-thermal-expansioninduced internal stresses. Since the microcracking behavior for both the strong $(R=1.0)$ and the weak $(R=0.4)$ grain boundary cases shows the same qualitative dependence on misfit 


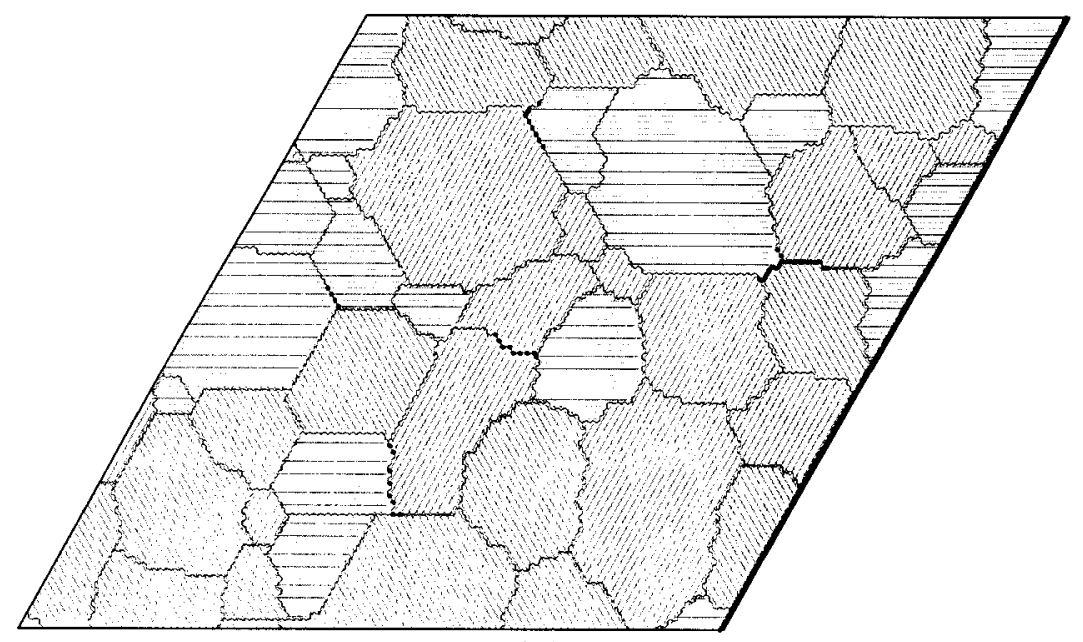

(a)

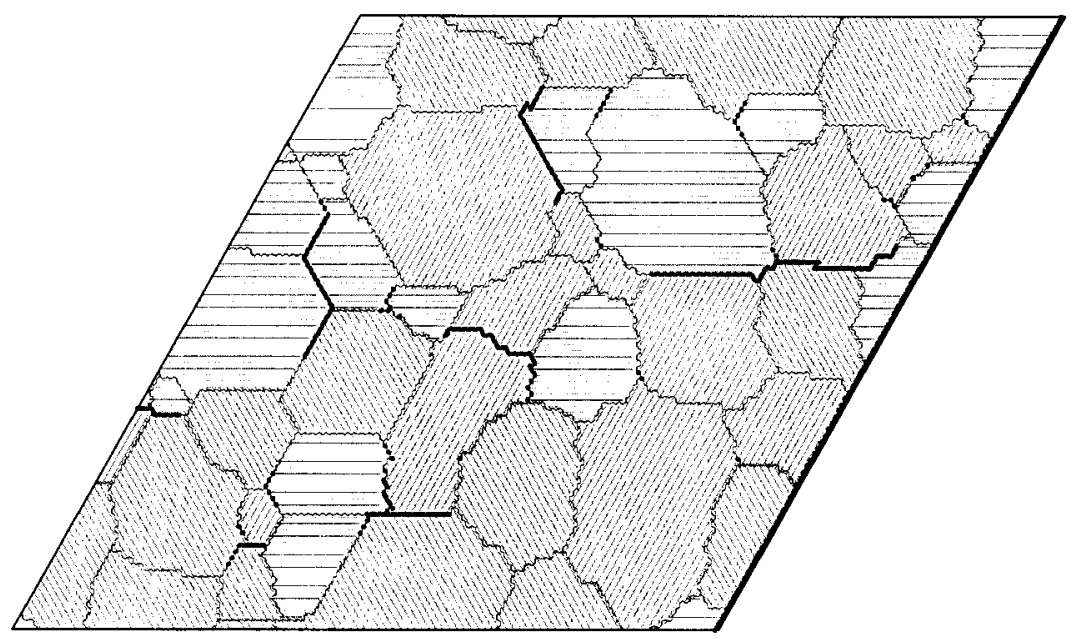

(b)

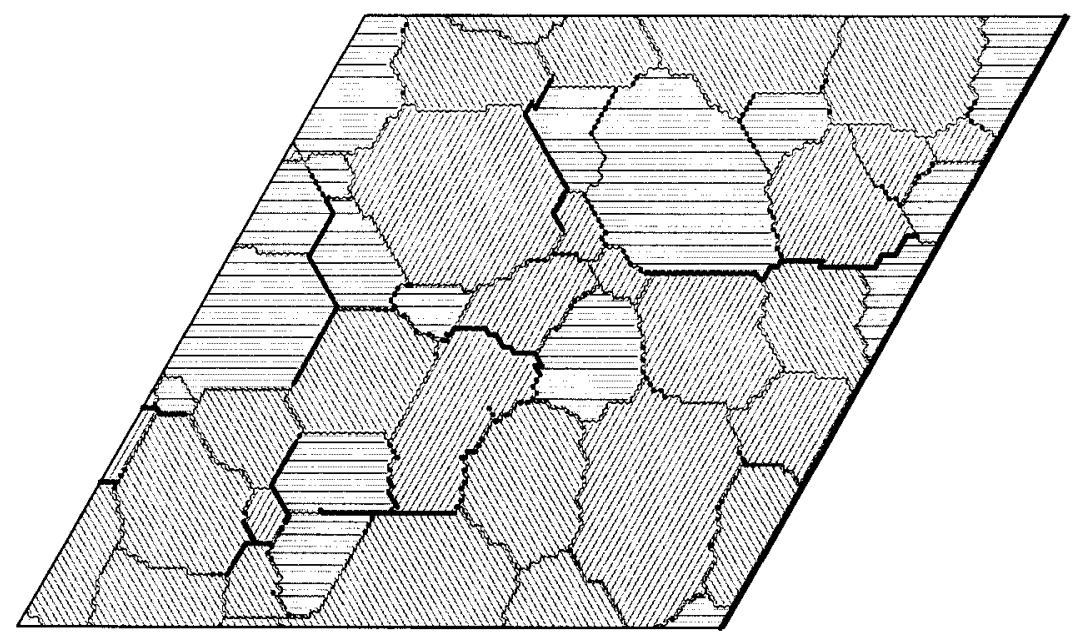

(c)

Fig. 6. Microcracked polycrystalline microstructures for a series of increasing misfit strains: (a) $e=0.0095$, (b) $e=0.0120$, and (c) $e=0.0150$. The grain size is $r=14.4$ (in units of the unstrained spring length) and the ratio of grain boundary to grain interior toughness is $R=0.4$.

strain and grain size, we present simulation results here for only the strong grain boundary case.

Figure 10 shows three microcracked microstructures with different grain sizes at a relatively small misfit strain of $e=$ 0.0095 . The smallest grain size $(r=4.5)$ microstructure (Fig. $10(\mathrm{a}))$ exhibits few broken bonds at this strain. In all cases, these microcracks form at or near grain boundaries. The intermediate grain size $(r=7.8)$ sample (Fig. 10(b)) shows many more broken bonds than does the small grain size sample at the same misfit strain. In this case, the cracks are substantially larger, are not necessarily at grain boundaries, and tend to run in a direction approximately normal to the constraint. The largest 


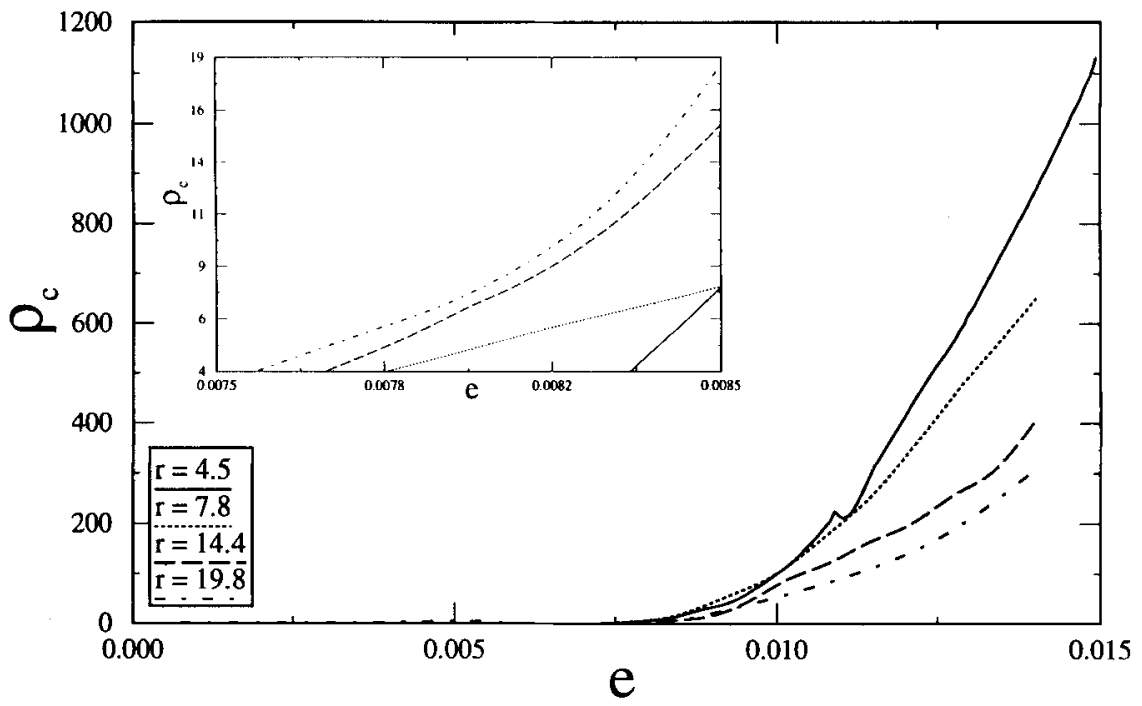

Fig. 7. Total number of broken bonds in the model $\rho_{\mathrm{c}}$ as a function of misfit strain $e$ for several different grain sizes in microstructures for which the grain boundary and bulk toughnesses are equal $R=0.4$. The inset shows the small misfit strain data in more detail.

grain size $(r=19.8)$ microstructure (Fig. 10(c)) shows several small cracks and one large crack that spans $70 \%$ of the sample width. As in the intermediate grain size case, these cracks tend to run in the direction normal to the constraint. Figure 11 shows the number of broken bonds as a function of the misfit strain for four different grain sizes. At small misfit strains, we observe (see the inset in Fig. 11) that the number of broken bonds is largest for the large grain size sample and smallest for the small grain size sample. Microcrack initiation occurs at $e=0.0072$ for the largest grain size sample whereas it occurs only at $e=$ 0.0085 for the smallest grain size sample. The main difference between the constrained and unconstrained samples is the presence of long continuous cracks under constraint, as compared with the large number of small cracks in the small grain size sample. The fact that these long cracks are oriented perpendicular to the constraint is attributable to the uniaxial tensile stress that develops in that direction.

Comparison of the microcracking behavior in the constrained and unconstrained cases (cf. Figs. 3 and 11) shows that the presence of the constraint does not change most of the microcracking features observed in the unconstrained microstructure. In particular, we note that at small misfit strains, there is more microcracking in the large grain size samples as compared to the small grain size samples (refer to inset of Figs. 3 and 11), while at large misfit strains, the microcrack density is smallest for the large grain size samples. However, when a constraint is imposed, there is an initial, rapid rise in the number of broken bonds followed by a slower increase with increasing misfit strain that is reminiscent of the $\rho_{c}$ versus $e$ plots when no constraint is applied (Fig. 3). This initial rapid rise is attributable to the directed microcracking attributable to the uniform stress associated with the constraint. This additional uniaxial stress also results in a lowering of the minimum misfit strain for microcracking.

\section{Discussion}

The results presented above show the variation of microcrack density with grain size and anisotropic-thermal-expansioninduced misfit strain (see Figs. 2 and 6). These misfit strains cause stresses that vary through the microstructure due to the grain by grain variation in the high thermal expansion direction and, hence, vary on a length scale comparable to the grain size. When the local, anisotropic-thermal-expansion-induced stresses are of sufficient magnitude to exceed the local failure condition, a microcrack forms and grows. In the present model, the degree of microcracking has been analyzed in terms of the total number of broken bonds, $\rho_{c}$, which is the product of the number of microcracks and the average microcrack length.

Examination of the effect of grain size on microcracking, we find two distinct classes of behavior. The first operates when the misfit strains are relatively small. In this regime, $\rho_{\varepsilon}$ is large for the large grain samples and relatively small in the small grain samples. In addition to having the largest $\rho_{c}$, the large grain size microstructures also exhibited longer cracks than did the small grain size microstructures. Prior to the beginning of microcracking, the samples with the largest grain size exhibit the largest internal stresses. Since microcracking begins when a critical stress or strain energy is exceeded, this explains why the larger grain size microstructures exhibit a larger $\rho_{c}$ at small misfits or $|\Delta T|$. The observed large crack length dependence on the grain size is attributable to the coherence length of the stress (i.e., the length scale over which the stress varies), which scales directly with the grain size.

When the misfit strains are large, a different, second class of behavior is observed. In this regime, $\rho_{c}$ is largest for the microstructures with the smallest grain size. Nonetheless, the longest microcracks are still found in the microstructures with the largest grain size. There are two possible explanations for the presence of more extensive microcracking in the small grain samples, as compared with the large grain samples. The first is associated with the fact that the formation of a large crack relieves stresses in a large area (in 2-d) or volume (in 3- $d$ ) surrounding it, where $d$ is dimensionality of the material. In fact, the region of stress relief scales as $a^{d}$, where $a$ is the crack length. The presence of long cracks in the large grain samples effectively prevents the formation and/or growth of additional microcracks in their immediate vicinity, as may be seen, for example, in Fig. 5(c). Since $a$ is proportional to the grain size $r$ and the stress relief area/volume scales as $a^{d}$, we expect that the total crack length/area (i.e., the number of broken bonds) per grain should scale as the grain size $r^{d \cdots 1}$. This further suggests that the total crack length/area per unit area/volume of the material, $\rho_{c}$, should scale inversely with the grain size, $\rho_{c} \propto 1 / r$. The second approach is based upon the observation that microcracks tend to form almost exclusively along grain boundaries. This is because the discontinuity in the misfit that occurs at grain boundaries leads to the formation of the largest stresses at the boundaries. This suggests that the number of microcracks per grain scales in proportion to the length/area of the grain boundaries per grain, i.e., $r^{d-1}$. In this case, all of the cracks cannot grow to a length comparable with the grain size because of the interaction between the regions of stress relaxation. Therefore, assuming that the crack length is independent of 


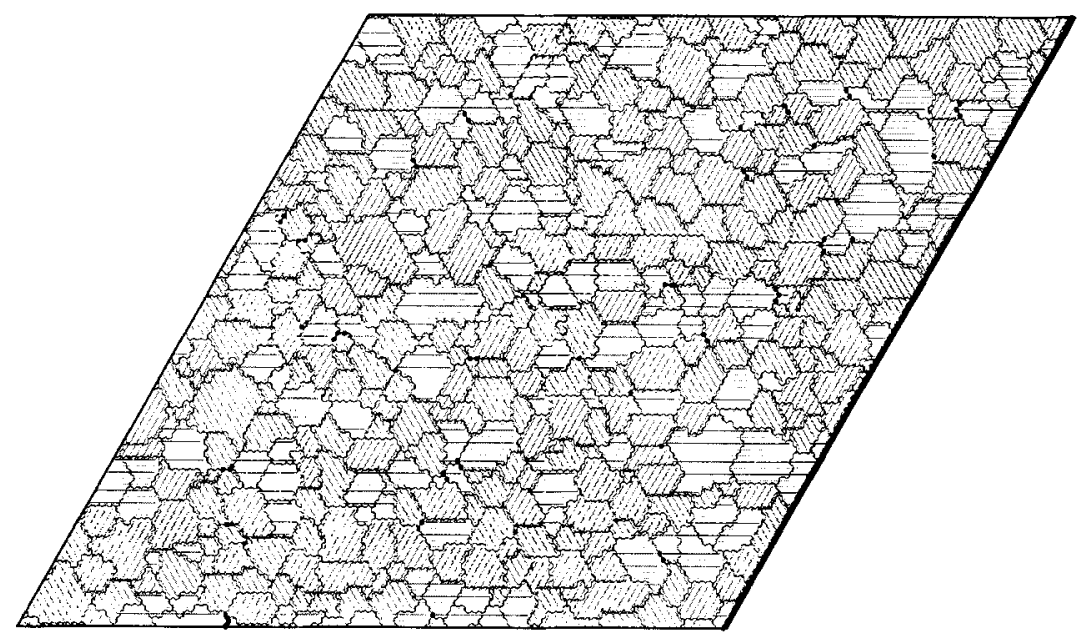

(a)

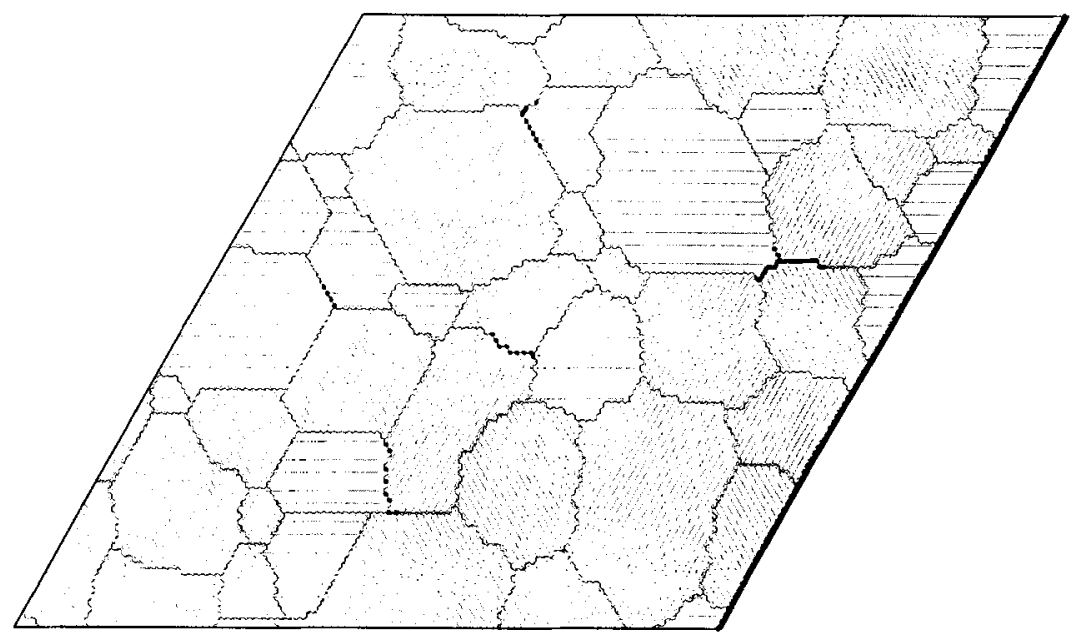

(b)

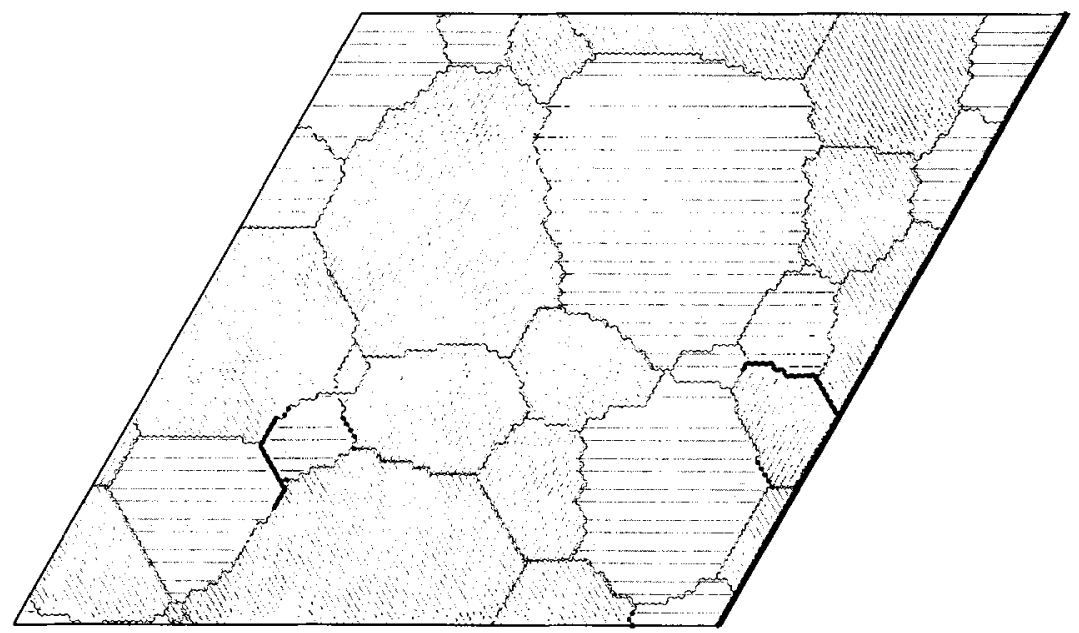

(c)

Fig. 8. Microcracked polycrystalline microstructures for a series of grain sizes: (a) $r=4.5$, (b) $r=14.4$, and (c) $r=19.8$. These results are for a small misfit strain $(e=0.0095)$ and the ratio of grain boundary to grain interior toughness is $R=0.4$.

grain size, we see that the total crack length per unit area/volume should again scale inversely with grain size, i.e., $\rho_{\mathrm{c}} \propto 1 / r$. While the assumption that the crack length is independent of grain size may not be valid, this second approach still leads to larger $\rho_{\mathrm{c}}$ for smaller grain sizes as long as the crack length scales with grain size more weakly than $a \propto r$ (e.g., $a \propto \ln r$ ). While the data presented above show that $\rho_{c}$ is largest for small grain microstructures at large misfit, in agreement with the above arguments, they are insufficient to unequivocally determine the functional form of $\rho_{c}(r)$.

The results presented above show the effect of variations in the grain boundary toughness relative to that of the grain interiors, $R$, on microcracking behavior. In short, when the grain boundaries are weak compared to the bulk (small $R$ ), micro- 


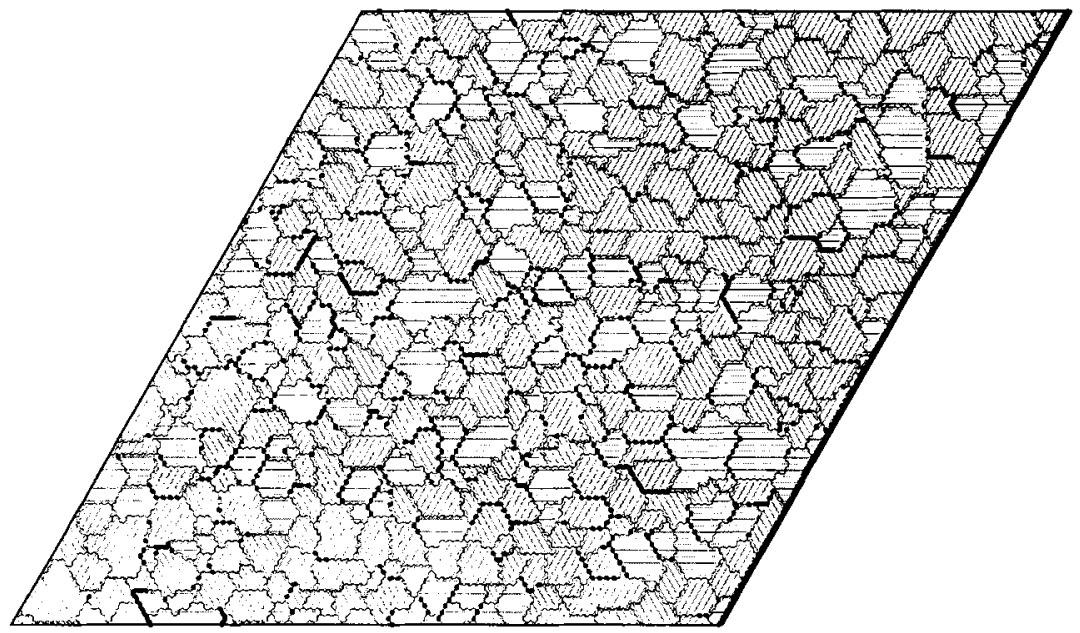

(a)

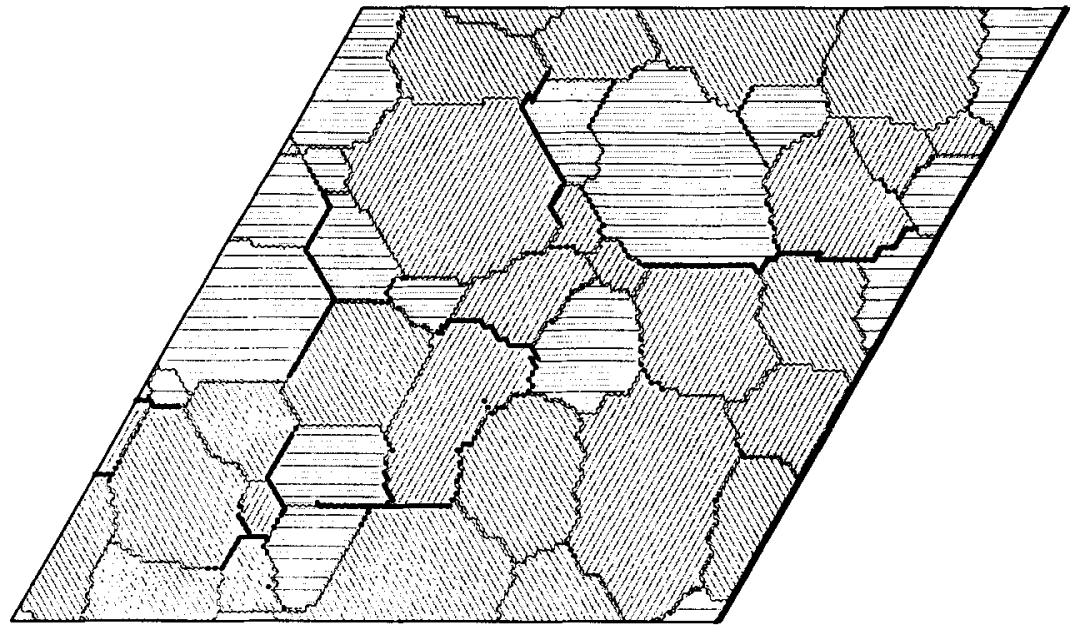

(b)

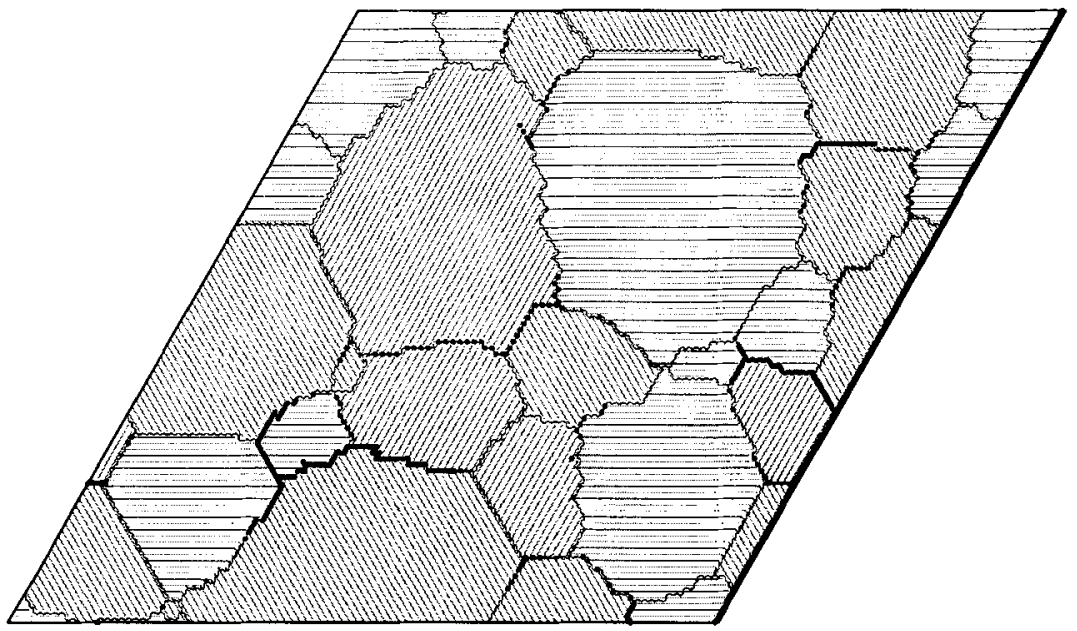

(c)

Fig. 9. Microcracked polycrystalline microstructures for a series of grain sizes: (a) $r=4.5$, (b) $r=14.4$, and (c) $r=19.8$. These results are for a large misfit strain $(e=0.0150)$ and the ratio of grain boundary to grain interior toughness is $R=0.4$.

cracking begins at the grain boundaries and further crack propagation is along the grain boundaries. When $R=1$, microcracking begins at the grain boundaries, but crack propagation tends to occur predominantly into the grain interiors. Therefore, reducing the grain boundary toughness simply shifts the microcracking behavior from transgranular to intergranular. Examination of the $\rho_{c}$ versus misfit strain plots (Figs. 3 and 7) shows that microcracking is more predominant in the large grain samples at small misfit and in the small grain samples at large misfit, independent of the relative grain boundary toughness, $R$. However, decreasing the grain boundary toughness at fixed grain interior toughness results in a decrease in the minimum misfit required for anisotropic-thermal-expansioninduced microcracking. 


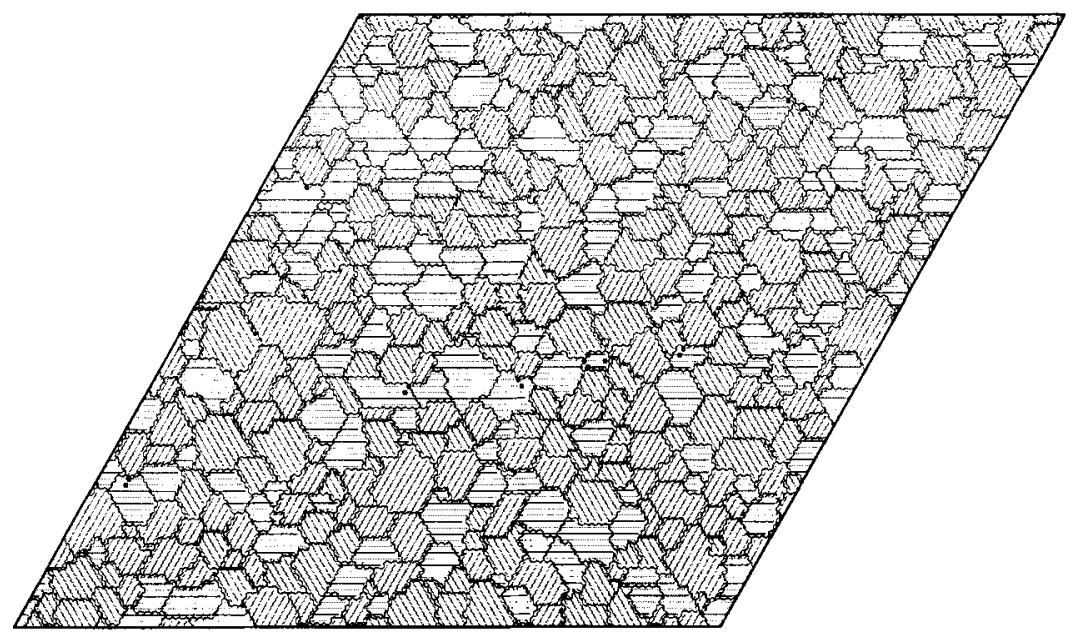

(a)

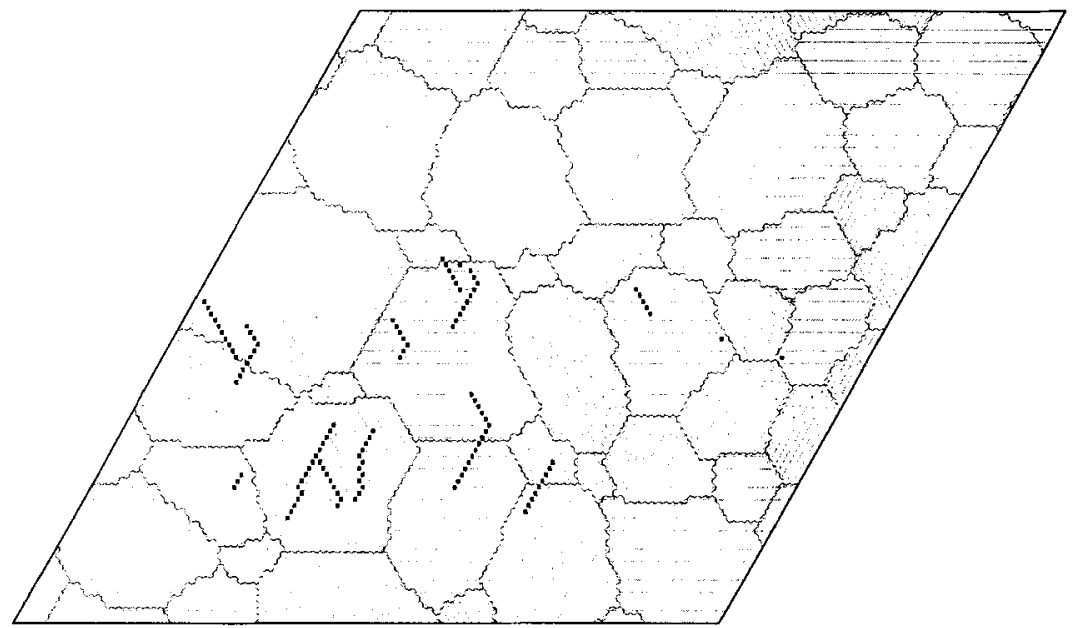

(b)

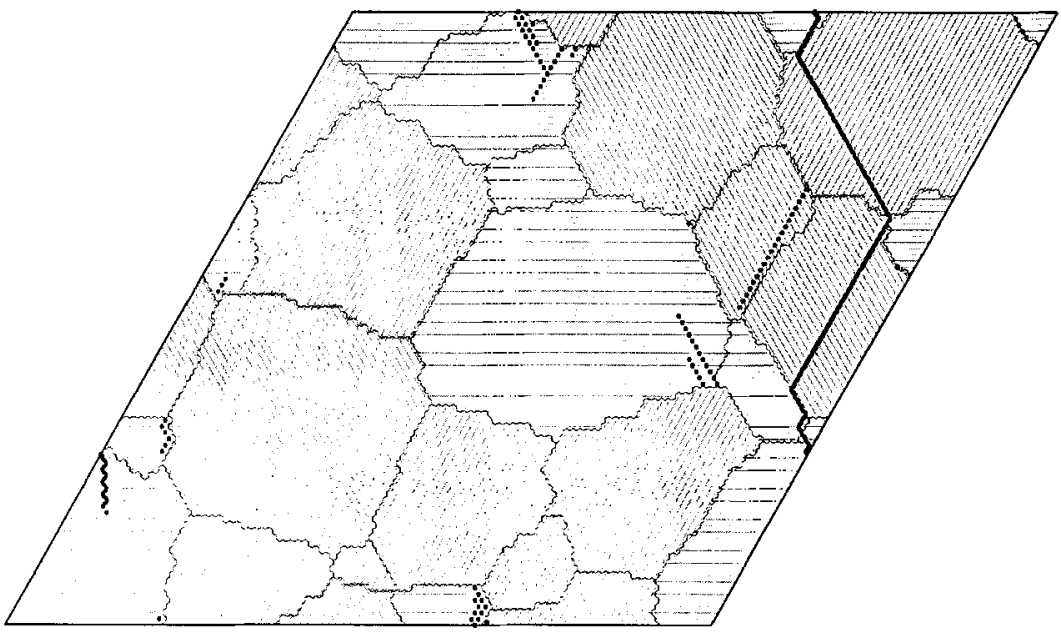

(c)

Fig. 10. Constrained microcracked polycrystalline microstructures for a series of grain sizes: (a) $r=4.5$, (b) $r=14.4$, and (c) $r=19.8$. The constraint is in the $x$-direction. These results are for a small misfit strain $(e=0.0095)$ and the ratio of grain boundary to grain interior toughness is $R=$ 1.0 .

While $\rho_{\mathrm{c}}$ provides a measure of the microcracking damage, it is not an easily accessible experimental parameter. Therefore, we also measured the uniaxial elastic modulus of the microcracked samples as a function of misfit strain. This was accomplished by heating (or cooling) the microcracked samples to their original misfit-stress-free temperature and then measuring their Young's modulus in uniaxial tension. No additional cracking occurred during these tensile tests. This procedure guaranteed that changes in the modulus were simply related to the microcracks and not to nonlinear elastic effects. Figure 12 shows the elastic modulus as a function of the misfit strain for both $R=0.40$ and $R=1.0$ as a function of the misfit strain that 


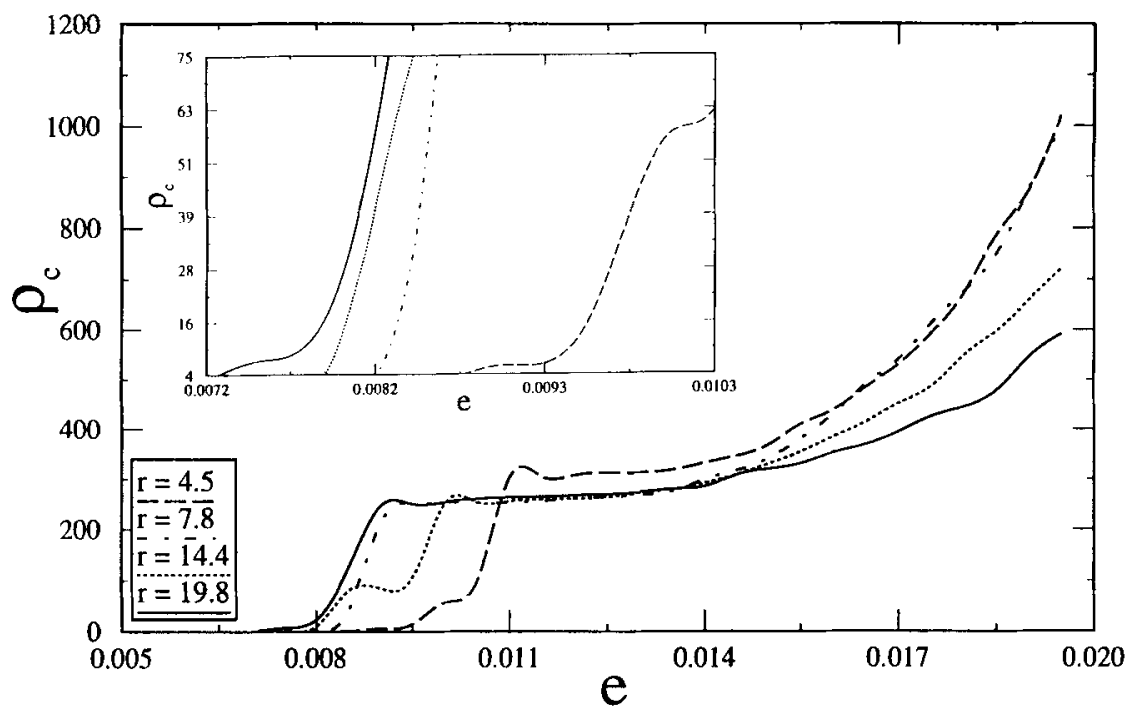

Fig. 11. Total number of broken bonds in the model $\rho_{\mathrm{c}}$ as a function of misfit strain $e$ for several different grain sizes in constrained samples for which the grain boundary and bulk toughnesses are equal, $R=1.0$. The inset shows the small misfit strain data in more detail.

originally produced the microcracked microstructures. Even though each curve represents the average of six independent simulations, there is still considerable statistical noise. Young's modulus for each sample decreases monotonically with increasing misfit strain and damage. When the misfit is large, the smaller grain size microstructures show a faster drop in the modulus than do the larger grain size samples with increasing misfit. Therefore, the modulus decrement shows the same general behavior with misfit strain as does the number of broken bonds $\rho_{\mathrm{c}}$ reported above.

Analytical models of the modulus decrement due to microcracking $^{18}$ suggest that the modulus decrement scales as the product of the crack density and the volume/area of the stress relieved region around each crack (i.e., the cube or square of the crack length in 3-d or 2- $d$, respectively). This prediction corresponds to the modulus decrement scaling as the product of the number of broken bonds per unit volume and the crack length. Figure 13 shows Young's modulus for the unconstrained microstructures as a function of the broken bond density $f$, obtained over a range of misfits (temperatures) and both grain boundary toughnesses examined above (i.e., $R=0.4$ and 1.0). All of the data fall into a fairly tight band, which on average decreases linearly with increasing fraction of broken bonds (i.e., $1-f$ ). This is consistent with previous theoretical results. ${ }^{18}$ The scatter in the data is attributable, in part, to the crack size dependence of the modulus. However, the limited range of crack sizes examined and the inherent statistical noise in the data prevent us from examining this prediction in more detail. Previous simulations ${ }^{19}$ of this type in which bonds were broken at random also showed a linear dependence of the modulus decrement on the broken bond density for broken bond densities not too near the rigidity percolation threshold. However, this earlier study showed a bond density coefficient notably larger than the present one. This is presumably attributable to the much smaller crack size (single sites) in the earlier study ${ }^{19}$ and the predicted dependence of the modulus decrement on crack size. ${ }^{18}$

While both Young's modulus and the total number of broken bonds provide a measure of microcracking damage, perhaps a more important measure is the influence of microcrack damage on the overall fracture behavior of the sample. Therefore, we have performed uniaxial tension tests (strain controlled) on the same microstructures as used for the Young's modulus measurements. Figure 14 shows the uniaxial stress strain curves for both a tough $(R=1.0$, Fig. 14(a) $)$ and weak $(R=0.4$, Fig. 14(b)) grain boundary microstructure. In both cases, the misfit strains were sufficiently large that $\rho_{c}$ was largest for the

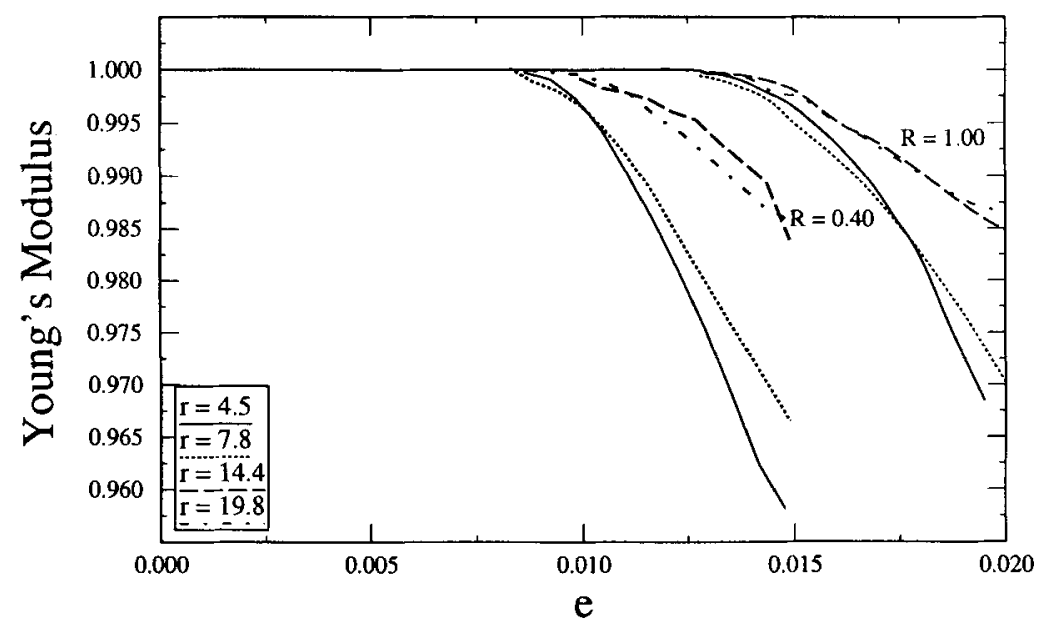

Fig. 12. Young's modulus of the microcracked microstructures as a function of the misfit strain responsible for the damage. Data are shown for several grain sizes and two values of the grain boundary toughness. The modulus is normalized to that of the uniform, damage-free microstructure. These data are for unconstrained microstructures. 


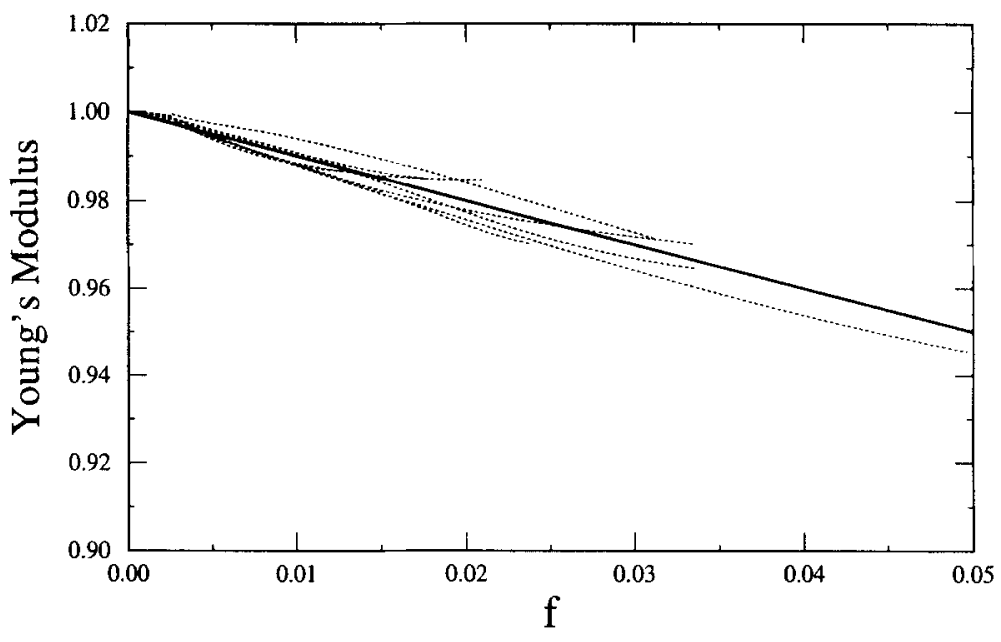

Fig. 13. Young's modulus of the microcracked microstructures as a function of the fraction of bonds that are broken $(f)$ during a temperature excursion. The narrow lines correspond to the unconstrained, microcracked microstructures with both weak $(R=0.4)$ and tough $(R=1.0)$ grain boundaries, presented in Section III(1). The wide line corresponds to the predicted Young's modulus dependence on the microcrack density (i.e., $1-f$ ).

small grain size microstructures. Nonetheless, Fig. 14 shows that the fracture stress is smallest for the largest grain size microstructure. Linear elastic fracture mechanics suggests that the fracture stress $\sigma_{\mathrm{f}}$ is proportional to the inverse square root of the crack length $\left(\sigma_{f} \propto a^{-1 / 2}\right)$ such that the microstructure with the longest crack is expected to fracture at the smallest externally applied stress. Since crack lengths in the anisotropicthermal-expansion-damaged microstructures scale with the grain size, the sample with the largest grain size is expected to fail at the lowest applied stress-in agreement with the present simulation results (Fig. 14).

When the surfaces of the sample are constrained during an anisotropic misfit strain inducing temperature excursion, an additional stress is superimposed upon the anisotropic-thermalexpansion-induced stresses present in an unconstrained sample. This additional stress does not vary on the same length scale as the anisotropic-thermal-expansion-induced stress (i.e., the grain size), but is superimposed upon the entire sample. This additional stress has two main effects: (1) it decreases the magnitude of the misfit strain necessary to induce microcracking and (2) it determines the direction of maximum crack growth. The fact that the cracks tended to propagate in the direction normal to the constraint suggests that the constraint stress dominates the anisotropic-thermal-expansion-induced stresses.

A large body of experimental evidence ${ }^{1-10.20 .21}$ suggests that large grain size microstructures are more susceptible to anisotropic-thermal-expansion-induced damage than are small grain microstructures. The present results show that the effect of grain size on anisotropic-thermal-expansion-induced microcracking can be greater for either large or small grain size samples depending on the measure employed. At all but the smallest misfits, the total length/area of all cracks in a sample will be larger when the grain size is small. This is manifested in the larger decrement of the elastic modulus in small grain size samples as compared with large grain size samples at the same misfit $(\Delta T)$. However, for a given misfit or temperature excursion, large grain sizes are more detrimental to overall fracture properties. This is because the fracture stress scales as the inverse square root of the crack length and large grain samples have larger anisotropic-thermal-expansion-induced microcracks than small grain samples. If a sample is rigidly constrained during the temperature excursion and the constraint stress exceeds the fracture stress of the anisotropic-thermalexpansion-microcracked sample, the temperature excursion can direcly lead to fracture of the entire sample. Experimentally, such constraints can be associated with the fixture holding the sample or, more commonly, due to macroscopic nonuniformities in the sample shape.
The simulation procedure used in the present study is based upon a discrete elastic model. As a result of this discretization, there are some genuine differences between the predictions of the model and that due to a continuum elastic analysis. One prime difference is the fact that the crack tip field is singular in a continuum elastic analysis, while it remains discrete here. In all real materials, the crack tip field singularity is relaxed by some type of dissipative means (e.g., plasticity, damage) or, in the most extreme cases, by the inherent atomic level discreteness of solids. The important point is that fracture mechanics is still valid no matter at what level the maximum stresses are limited. The maximum stress near a crack tip seen in the present model is set by our choice of discreteness, i.e., $a_{0}$. Since this limit is set arbitrarily, the maximum stress near a crack tip in the present model has no physical meaning. Therefore, while the model does accurately describe the elastic field of a crack a few $a_{0}$ away from the tip, it cannot be relied upon to give physically meaningful values of parameters such as fracture stress, critical misfit strain, etc. Fortunately, this is not a problem since the purpose of the present study is not to predict fracture stresses, but simply to examine the effect and trends associated with varying microstructural features. A similar discreteness question arises when discussing grain size effects. As long as the grain size in the simulation is much larger than the spring size (i.e., $r>>a_{0}$ ), the discreteness of the model should have little effect on the trends in microcracking behavior. Nonetheless, the discreteness does render a quantitative comparison of grain size effects in simulation and experiment meaningless. However, trends in microcracking behavior, as opposed to absolute values, should still be reliable.

The present simulations were performed on a two-dimensional model, although essentially all experiments have an important three-dimensional component. Strictly speaking, the present results should be applied only to the analysis of thermal-expansion-anisotropy-induced microcracking in freestanding (no substrate) thin films where the average grain size is larger than the film thickness. Nonetheless, we expect that the microcracking trends observed in the present study will also apply to three-dimensional samples. In particular, we are referring to the presence of a critical grain size for microcracking, the larger degree of microcracking in large grain samples for small misfit strains, the larger degree of microcracking in small grain samples for large misfit, the more severe effect of microcracking on overall fracture behavior in large grain samples than in small grain ones, etc. However, the detailed functional form of the dependence of some of the physical parameters (e.g., modulus change) on grain size may be explicitly dependent on dimension and hence the functional forms may not be reliable. 


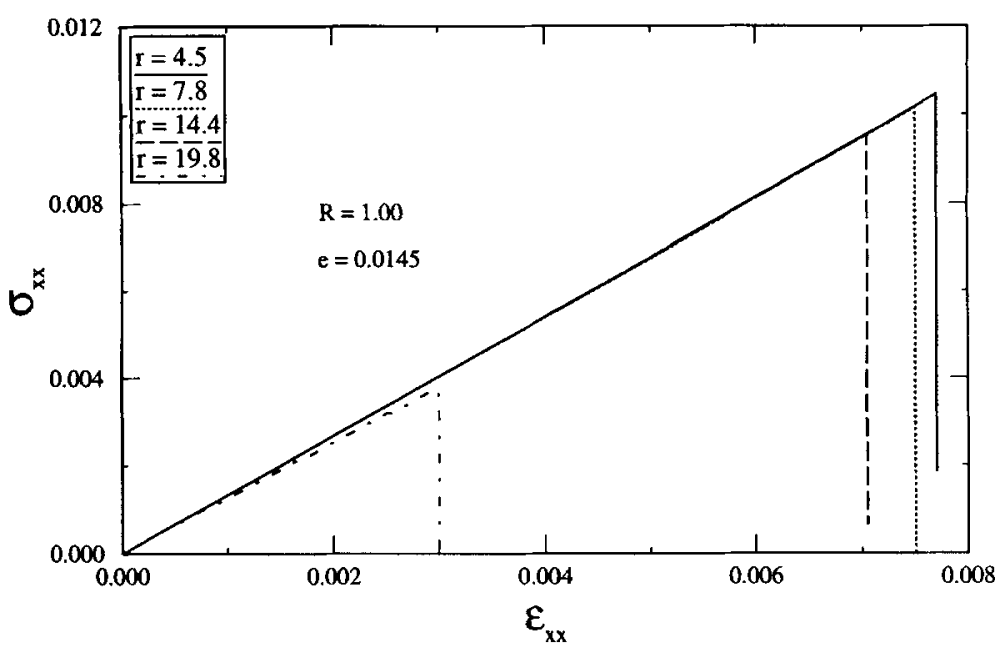

(a)

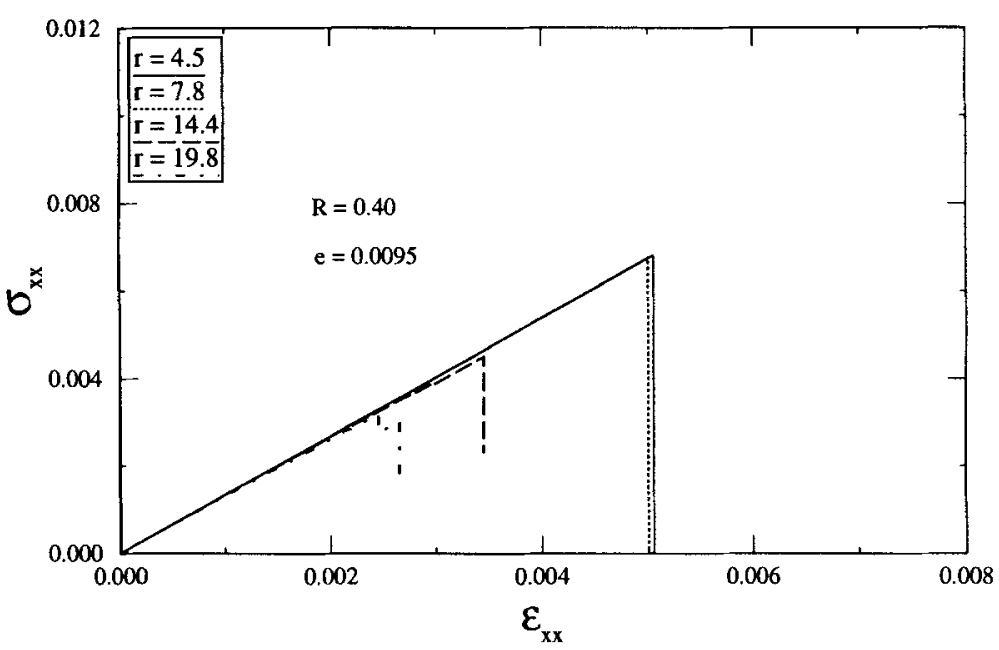

(b)

Fig. 14. Stress-strain curves for unconstrained, microcracked microstructures for several grain sizes: (a) results for microstructures with tough grain boundaries $(R=1.0)$ and a misfit strain of $e=0.0145$; (b) results for microstructures with weak grain boundaries $(R=0.4)$ and a misfit strain of $e=$ 0.0095 . These data were not averaged over multiple simulation runs.

Given these caveats, we can still compare our simulation results with the large body of experimental literature on thermal-expansion-induced microcracking, although only qualitatively. Kuszyk and $\mathrm{Bradt}^{2}$ reported that a noncubic ceramic $\left(\mathrm{MgTi}_{2} \mathrm{O}_{5}\right)$ with a large thermal expansion anisotropy shows an abrupt transition in fracture strength and elastic modulus as a function of grain size. These results are consistent with those reported above; namely, the microcrack density and hence the fracture strength and moduli are sensitive functions of the grain size (see Figs. 3, 7, 12, 14). Several experimental studies and fracture mechanics analyses ${ }^{7,10-13}$ reveal that the critical grain size for onset of microfracture is proportional to the inverse square root of the maximum thermal expansion difference (i.e., $\left.r_{\mathrm{c}} \propto(\Delta \alpha)^{-2}\right)$. The dependence of the critical grain size on thermal expansion mismatch, extracted from the above results, yields $r_{c} \propto(\Delta \alpha)^{-\beta}$, where $-1>\beta>-2$ (data extracted from Figs. 3 and 7). This range of $\beta$ values is reasonably close to that reported in other works, given the poor statistics in the present determination of $\beta$. Both a large body of experimental results and the present simulations show that microcracking is especially large in cases where the grain boundaries separate adjoining grains with large thermal expansion mismatch. Bush and Hummel $^{22.23}$ reported a modulus drop with an increase in the microcrack density. This is consistent with our observations (see Fig. 12). Finally, we note that Kuszyk and Bradt ${ }^{2}$ found substantially lower fracture stresses in large grain samples than in small grain ones, also in agreement with our present results (see Fig. 14). Therefore, the present simulation results do reproduce much of the qualitative experimental observations of thermal-expansion-anisotropy-induced microcracking and its effect on the mechanical and fracture behavior of ceramics. However, direct quantitative comparison is not possible.

\section{References}

'J. P. Singh, D. P. H. Hasselman, W. M. Su, J. A. Rubin, and R. Palicka, "Observations on the Nature of Micro-cracking in Brittle Composites,"J. Mater. Sci., 16, 14l-50 (1981).

${ }^{2}$ J. A. Kuszyk and R. C. Bradt, "Infuence of Grain Size on Effects of Thermal Expansion Anisotropy in $\mathrm{MgTi}_{2} \mathrm{O}_{5}, " J$. Am. Ceram. Soc, 56, 420-23 (1973).

${ }^{3}$ A. G. Evans and K. T. Faber, "Crack-Growth Resistance of Microcracking Brittle Materials," J. Am. Ceram. Soc., 67, 255-60 (1984).

${ }^{4} \mathrm{~V}$. Tvergaard and J. W. Hutchinson, "Microcracking in Ceramics Induced by Thermal Expansion or Elastic Anisotropy," J. Am. Ceram. Soc., 71, 157-66 (1988).

${ }^{5}$ E. H. Lutz, N. Claussen, and M. V. Swain, " $K^{R}$-Curve Behavior of Duplex Ceramics,"J. Am. Ceram. Soc., 74, 11-18 (1991).

${ }^{6} \mathrm{~J}$. F. Quirk, N. B. Mosley, and W. H. Duckworth, "Characterization of Sinterable Oxide Powders: I, BeO," J. Am. Ceram. Soc., 40, 416-19 (1957).

${ }^{7}$ F. J. P. Clarke, "Residual Strain and the Fracture Stress-Grain Size Relationship in Brittle Solids," Acta Metall, 12, 139-43 (1964).

${ }^{8}$ R. W. Rice and R. C. Pohanka, "Grain-Size Dependence of Spontaneous Cracking in Ceramics," J. Am. Ceram. Soc., 62, 559-63 (1979).

${ }^{9}$ R. W. Rice, S. W. Frieman, and P. F. Becher, "Grain-Size Dependence of Fracture Energy in Ceamics: I, Experiment; II, A Model for Noncubic Materials," J. Am. Ceram. Soc., 63, 345-54 (1981). 
"A. G. Evans, "Microfracture from Thermal Expansion Anisotropy-I. Single Phase Systems," Acta Metall., 26, 1845-53 (1978).

'D. R. Clarke, "Microfracture in Brittle Solids Resulting from Anisotropic Shape Changes," Acta Metall., 28, 913-24 (1980).

${ }^{12}$ A. G. Evans and Y. Fu; p. 154 in Fracture in Ceramic Materials. Edited by A. G. Evans. Noyes, Park Ridge, NJ, 1984.

${ }^{3} \mathrm{R}$. W. Davidge, "Cracking at Grain Boundaries in Polycrystalline Materials," Acta Metall., 29, 1695-702 (1981).

${ }^{14}$ W. H. Yang, D. J. Srolovitz, G. N. Hassold, and M. P. Anderson, "Microstructural Effects in the Fracture of Brittle Materials"; pp. 277-84 in Simulation and Theory of Evolving Microstructures. Edited by M. P. Anderson and A. D. Rollett. The Metallurgical Society, Warrendale, PA, 1990.

${ }_{15}^{15}$ J. F. Nye, Physical Properties of Crystals. Clarendon Press, Oxford, U.K., 1985.

${ }^{16}$ D. J. Srolovitz, M. P. Anderson, P. S. Sahni, and G. S. Grest, "Computer Simulation of Grain Growth: II. Grain Size Distribution, Topology and Local Dynamics," Acta Metall., 32, 793-802 (1984).
${ }^{17}$ G. S. Grest, M. P. Anderson, and D. J. Srolovitz, "Computer Simulation of Normal Grain Growth in Three Dimensions," Philos. Mag., B59, 293-329 (1988).

"B. Budiansky and J. O'Connell, "Elastic Moduli of Cracked Solids," Int. J. Solids Struct., 12, $81-97$ (1976).

${ }^{19}$ D. J. Srolovitz and P. D. Beale, "Computer Simulation of Failure in an Elastic Model with Randomly Distributed Defects," J. Am. Ceram. Soc., 71, 362-69 (1988).

${ }^{20}$ Y. Ohya, Z. Nakagawa, and K. Hamano, "Grain-Boundary Microcracking Due to Thermal Expansion Anisotropy in Aluminium Titanate Ceramics," J. Am. Ceram. Soc., 70, 184-86 (1987).

2'J. J. Cleveland and R. C. Bradt, "Grain Size/Microcracking Relations for Pseudobrookite Oxides," J. Am. Ceram. Soc., 61, 478-81 (1987).

${ }^{22}$ E. A. Bush and F. A. Hummel, "High Temperature Mechanical Properties of Ceramic Materials: I," J. Am. Ceram. Soc., 41, 189-95 (1958).

${ }^{23}$ E. A. Bush and F. A. Hummel, "High Temperature Mechanical Properties of Ceramic Materials: Il," J. Am. Ceram. Soc., 42, 388-91 (1959). 